\title{
Potencial de Biomasa en América del Sur para la Producción de Bioplásticos. Una Revisión
}

\author{
Vargas-García, Yadira 1, 2, 3, * (D) ; Pazmiño-Sánchez, Joffre ${ }^{4}$; ; Dávila-Rincón, Javier ${ }^{5}$ (D) \\ ${ }^{I}$ Instituto Superior Tecnológico Crecermás, Lago Agrio - Ecuador \\ ${ }^{2}$ Pontificia Universidad Católica del Ecuador, Sede Amazonas, Lago Agrio - Ecuador \\ ${ }^{3}$ Universidad de Jaén, Campus las Lagunillas, Departamento de Ingeniería Química, Ambiental y de los Materiales; Campus de \\ Excelencia Internacional Agroalimentario (ceiA3), Jaén, España \\ ${ }^{4}$ Soluciones Profesionales Biobasados e Industrialización Limpia - SOPBIAL Cía. Ltda., Dirección de proyectos, Lago Agrio- \\ Ecuador \\ ${ }^{5}$ Universidad de Bogotá Jorge Tadeo Lozano, Departamento de Ingeniería, Bogotá - Colombia
}

\begin{abstract}
Resumen: La biomasa es una fuente primaria renovable que ha generado mayor interés en el mundo durante los últimos años, debido a la oportunidad de obtener productos energéticos y no energéticos similares a los que se producen de las fuentes no renovables. En este sentido, América del Sur, por su ubicación geográfica, cuenta con alto potencial de generar y adicionar valor a la biomasa; por lo que esta investigación realiza una revisión descriptiva sobre la disponibilidad y potencial de biomasa en América del Sur, con el propósito de establecer los tipos y cantidad de bioplásticos biobasados y biodegradables (BBB) que se pueden producir en esta región. En primer lugar, se realizó una descripción de la biomasa, sus características y clasificación. Posterior a esto, se recopiló información de la biomasa generada en cada país, para finalmente describir los tipos de BBB existentes en la actualidad. La investigación reveló que en la región se pueden producir 204,42 millones de toneladas (MMt) de bioplástico basado en celulosa regenerada, 4,11 MMt de bioplástico basado en almidón, 0,58 MMt de ácido poliláctico y 8,68 MMt de polihidroxibutirato. Además, la producción de $\mathrm{BBB}$ es una alternativa que permite minimizar la contaminación ambiental producida por los plásticos sintéticos, ya que utiliza de manera eficiente y sostenible los residuos generados por actividades agropecuarias e industriales. Finalmente, este estudio permite ampliar el estado del arte e intensificar las ventajas que tienen los países de América del Sur con respecto a la generación de empleos y desarrollo industrial en zonas rurales.
\end{abstract}

Palabras clave: Bioeconomía, bioindustrias, biomasa, plásticos biobasados y biodegradables.

\section{Biomass Potential in South America for the Production of Bioplastics. A review}

\begin{abstract}
Biomass is a primary renewable source that has generated greater interest in the world in last years, because it offers the opportunity to obtain energy and non-energy products similar to those manufactured from non-renewable sources. In this sense, South America, due to its geographical location, has high potential to generate and add value to biomass; so that this research carries out a descriptive review on the availability and potential of the biomass in South America, with the purpose of establishing the types and quantity of biobased and biodegradable bioplastics (BBB) that can be produced in this region. In the first place, a description of the biomass, its characteristics and classification was made. Subsequently, information was collected on the biomass generated in each country, to finally describe the types of BBB currently available. The research revealed that 204.42 million of tons (MMt) of bioplastic based on regenerated cellulose can be produced in the region, 4.11 MMt of starch-based bioplastic, $0.58 \mathrm{MMt}$ of polylactic acid and 8.68 MMt of polyhydroxybutyrate. In addition, the production of BBB is an alternative that allows to minimize the environmental pollution produced by synthetic plastics, since it uses in an efficient and sustainable way the waste generated by agricultural and industrial activities. Finally, the study made it possible to expand the state of the art and intensify the advantages that the countries of South America have with respect to the generation of jobs and industrial development in rural areas.
\end{abstract}

Keywords: Bioeconomy, bioindustries, biomass, biobased and biodegradable plastics.

\section{INTRODUCCIÓN}

En la última década el uso de la biomasa creció significativamente, destinándose a la producción de bioenergía y materiales biobasados (Gerssen-Gondelach et al., 2014). Además, en el año 2016, la biomasa ocupó el cuarto lugar en el consumo total de energía del mundo, precedido por el carbón, petróleo y gas natural. (Wang et al., 2016). Según 
investigaciones, esta materia prima renovable puede convertirse en una de las principales fuentes mundiales de energía primaria durante el presente siglo XXI (Berndes et al., 2003).

Las principales ventajas que tiene el uso de la biomasa como recurso energético en lugar del petróleo, carbón y gas, son la mejora de la situación socio-económica de las áreas rurales mediante el aprovechamiento de los residuos agrícolas y la reducción de las emisiones de azufre, partículas, $\mathrm{CO}, \mathrm{CH}_{4} \mathrm{y}$ $\mathrm{NO}_{\mathrm{x}}$ al medio ambiente. Además, la biomasa posee un ciclo neutro de $\mathrm{CO}_{2}$ que no contribuye al efecto invernadero (MEER, 2014). Varias investigaciones demuestran que América Latina y el Caribe (ALC) y África Subsahariana son potenciales productores de biomasa que podrían desempeñar un papel importante para el desarrollo de la bioeconomía (Gerssen-Gondelach et al., 2014).

Dentro de ALC se encuentra América del Sur, la cual representa el $42 \%$ del continente americano con una superficie de 18 millones de $\mathrm{km}^{2}$. En ella habitan alrededor del 6\% de la población mundial y posee una gran variedad de climas como húmedo-cálido, frío seco y húmedo, templado, mediterráneo, tropical y ecuatorial (CEPAL, 2008). América del Sur se caracteriza principalmente por la extracción de recursos naturales (mineros, petroleros y/o gas) y actividades agrícolas (PNUMA, 2013). Por otro lado, en el año 2019 esta región consumió el 4,90\% de la oferta mundial de energía primaria en las que se incluyen las energías renovables (BP, 2020). Asimismo, las emisiones de $\mathrm{CO}_{2}$ en este mismo año correspondieron al $3,7 \%$ de las emisiones mundiales relacionadas con la combustión de petróleo, gas y carbón (BP, 2020).

El petróleo mediante el proceso de refinación que se realiza en una refinería es separado en diversas fracciones, en las cuales se obtiene la nafta, que es el principal elemento para la producción de plásticos (PlasticsEurope, 2017). En América del Sur cada año se produce el $4 \%$ de la producción mundial de plásticos de un solo uso (ONU Medio Ambiente, 2018; PlasticsEurope, 2020). Sin embargo, el uso de estos materiales ha contribuido en gran medida a la contaminación ambiental, ya que una vez desechados pueden permanecer hasta 2.000 años en el ambiente ya sea en vertederos, lagos u océanos (Digregorio, 2009).

Los plásticos desempeñan un papel insustituible en la vida cotidiana de los seres humanos. Actualmente su consumo a nivel mundial es mayor al consumo propio del acero (Wang et al., 2016). En vista de esto, la industria del plástico enfrenta un gran desafío con el ambiente, desde la utilización de sus materias primas hasta los productos que se obtienen. Para lo cual, es de suma importancia buscar alternativas que garanticen el desarrollo sostenible de esta industria. En este sentido, una transición de la economía actual basada en combustibles fósiles a una economía basada en biomasa puede ser un camino óptimo a seguir (Sleenhoff et al., 2015; Wang et al., 2016).

Los bioplásticos son uno de los principales bioproductos que se comercializan dentro de la economía basada en la biomasa. Estos son fabricados a partir de materias primas renovables y/o biodegradables con una amplia gama de propiedades y aplicaciones (European Bioplastics, 2016). Según la Organización Europea de bioplásticos, estos materiales se encuentran divididos en tres grupos (European Bioplastics, 2016). En el primer grupo están los biobasados y no biodegradables como el polietileno, tereftalato de polietileno y poliamidas. El segundo grupo corresponde a los biobasados y biodegradables como el ácido poliláctico (PLA), polihidroxialcanoatos (PHA's), basados en almidón, celulosa y proteínas. Por último están los basados en recursos fósiles y biodegradables como el adipato de polibutileno tereftalato (European Bioplastics, 2016). Los bioplásticos tienen una historia de aproximadamente 150 años, estos materiales perdieron su importancia con el auge de la industria petroquímica en los años 50. Actualmente, el renacimiento de estos materiales ha sido impulsado por el progreso de la biotecnología y la preservación del ambiente (Pawelzik et al., 2013).

Cada año se produce cerca de 370 MMt de plásticos a nivel mundial (PlasticsEurope, 2020), de los cuales los bioplásticos representan apenas el $1 \%$, sin embargo, esta producción ha aumentado alrededor del $20 \%$ por año, debido al incremento de materiales con mejores características técnicas que se ofrecen en el mercado (European Bioplastics, 2017a). De acuerdo a esto, los bioplásticos podrían sustituir técnicamente alrededor del $85 \%$ de los plásticos convencionales en un mediano y largo plazo (European Bioplastics, 2017b).

Finalmente, tomando en cuenta lo mencionado anteriormente y con el fin de adicionar valor a la biomasa, la presente investigación abarca una revisión descriptiva, fundamentada en artículos científicos, organismos nacionales e internacionales que han sido publicados en los últimos 7 años detallando la disponibilidad de biomasa en los países de América del Sur, con el fin de establecer los tipos y la cantidad de bioplásticos biobasados biodegradables que se pueden producir en la región; esta cantidad es determinada a partir de los rendimientos establecidos por el Instituto de Bioplásticos y Biocompuestos. Consecuentemente, se inicia con una descripción general de la biomasa, para posteriormente detallar la disponibilidad de esta materia prima en América del Sur y finalmente puntualizar las perspectivas y clasificaciones de los BBB.

\section{BIOMASA}

La biomasa captura la energía del sol mediante el proceso de la fotosíntesis. Esta energía es transportada a través de los diferentes eslabones de la cadena alimentaria hasta llegar al ser humano, la cual es utilizada como materia prima para obtener diferentes bioproductos energéticos y no energéticos (Zhang \& Wang, 2013). En términos específicos, la biomasa es una fuente de energía renovable proveniente de los residuos de la materia orgánica, los cuales se generan principalmente de las actividades agrícolas, pecuarias, forestales, agroindustriales, acuosas, residuos urbanos y cultivos con fines energéticos (MEER, 2014). Las principales características que tiene esta fuente primaria son: carbono neutral, distribución geográfica relativamente uniforme, puede desarrollarse cerca de donde se usa, contribuye en gran medida al desarrollo socio-económico 
y es relativamente económica en comparación con las fuentes primarias no renovables (Sims \& Bassam, 2004).

En la Figura 1, se muestra la clasificación de la biomasa en función de su origen. Esta se encuentra dividida en biomasa residual y cultivos energéticos. La primera hace referencia a los subproductos que se derivan de las transformaciones naturales o industriales de la materia orgánica (MEER, 2014). Esta biomasa proviene de los residuos de madera, forestales, agrícolas, pecuarios, municipales y agroindustriales (Prando, 2015). Los residuos de madera comprenden los restos de aserríos como cortezas, astillas y aserrín. Mientras tanto, los residuos forestales son aquellos restos de plantaciones tales como ramas, cortezas y troncos. Los residuos agrícolas son los que se generan en las cosechas de los cultivos como: podas, tallos, cáscaras, raquis, etc. (MEER, 2014). Estos tres tipos de residuos forman parte de la biomasa lignocelulósica, cuyos principales componentes son: lignina (10-25\%), celulosa (40$80 \%)$ y hemicelulosa $(15-30 \%)$, las cuales varían por las características específicas de cada materia prima (Faba et al., 2013; Singh et al., 2017). Con respecto a los residuos pecuarios y municipales, estos corresponden a los residuos orgánicos de ciudad, excretas y purines (Bioenarea, 2013; Fabio et al., 2017). Por último, se encuentran los residuos agroindustriales que se generan cuando las materias primas agrícolas y ganaderas son utilizadas en procesos industriales por ejemplo: cáscaras, pulpas de frutas, bagazos, residuos de carne, lácteos, grasas y aceites vegetales y animales, entre otras (MEER, 2014; OLADE, 2017).

Mientras tanto, los cultivos energéticos son destinados principalmente para la obtención de bioenergía en forma de combustible sólido, líquido o gaseoso (Ambientum.com, 2015; Sims \& Bassam, 2004). Dentro de estos cultivos se encuentran los oleaginosos (colza, soja, palma aceitera, higuerilla, piñón, etc.), azucareros (sorgo dulce, remolacha azucarera, caña de azúcar, etc.) (Bioenarea, 2013; Sims \& Bassam, 2004) y acuosos, los cuales hacen referencia a las algas. Esta última se desarrolla en ambientes controlados mediante fotobiorreactores en áreas menos extensivas de terrenos (IICA, 2010). Tanto para la biomasa residual como para los cultivos energéticos, las composiciones físicas y químicas difieren entre sí, por lo que condiciona directamente las tecnologías de conversión y cada una de las etapas logísticas (Prando, 2015).

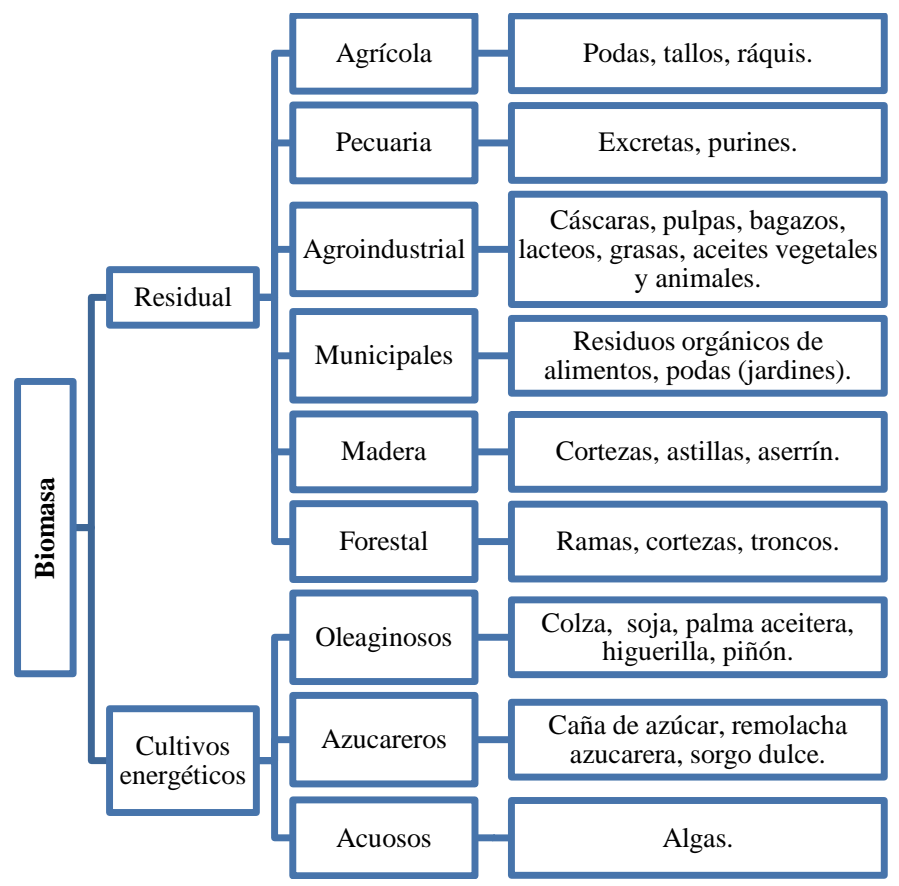

Figura 1. Clasificación de biomasa según su origen Adaptado de (Sims \& Bassam, 2004)

Adicionalmente, la biomasa se la puede clasificar en generaciones, es decir primera, segunda y tercera generación. La biomasa de primera generación hace referencia a los cultivos destinados a consumo humano tales como: el maíz, banano, soja, palma aceitera, arroz, entre otros; que por su misma naturaleza enfrentan diversos desafíos sociales, económicos y ambientales, ya que están totalmente relacionados con la seguridad alimentaria; además de causar problemas en el uso del suelo y generar un incremento de precios. La biomasa de segunda generación permite mayores posibilidades de sostenibilidad en comparación a la clasificación anterior, ya que corresponde a productos que no están relacionados directamente con el consumo humano, tales como: residuos domésticos e industriales, residuos sólidos municipales, residuos agrícolas, forestales y animales, así como también los cultivos energéticos como el piñón de tempate y la higuerilla, que representan riesgos a la salud de los humanos. La dificultad con la segunda clasificación se relaciona al mínimo desarrollo de rutas de conversión para obtener los bioproductos finales. Finalmente, la biomasa de tercera generación corresponde al aprovechamiento de algas y micro algas ya que son totalmente renovables y su potencial energético es mayor en comparación a las biomasas detalladas anteriormente; sin embargo, el desarrollo de sus procesos es incipiente y se requieren investigaciones de mayor avance científico (Pazmiño-Sánchez et al., 2017).

Por otro lado, la CEPAL (Comisión Económica para América Latina y el Caribe) clasifica a la biomasa como moderna y tradicional. La biomasa tradicional es la destinada a calefacción y preparación de alimentos en los hogares y la moderna está destinada a generación de electricidad, vapor, producción de biocombustibles y materiales biobasados (CEPAL, 2006). A su vez, clasifica el consumo de la biomasa en uso sostenible y no sostenible, dentro de la sostenible se incluyen residuos animales, vegetales, urbanos y leña 
recolectada de ramas secas y tala de árboles que serán replantados. La biomasa no sostenible está compuesta básicamente por la leña que proviene de la deforestación (CEPAL, 2006).

La biomasa contiene elementos químicos similares a los recursos no renovables como el petróleo, gas y carbón. De modo que, de esta materia prima se puede obtener una variedad de bioproductos energéticos y no energéticos, los cuales pueden sustituir parcial o completamente a los productos derivados de los recursos fósiles. Algunos de los bioproductos que se obtienen en la actualidad a partir de biomasa son: biocombustibles, biolubricantes, biopolímeros, pinturas, adhesivos, espesantes, estabilizadores, telas y una gama de celulósicos (Sims \& Bassam, 2004). Por otro lado, a diferencia de los combustibles fósiles, la combustión de la biomasa no contribuye al aumento de gases de efecto invernadero (GEI). Esto se debe a que el carbono que se libera es absorbido continuamente por la vegetación durante su crecimiento (MEER, 2014), es decir la emisión de $\mathrm{CO}_{2}$ es neutra (GerssenGondelach et al., 2014; OLADE, 2017).

\section{POTENCIAL DE BIOMASA EN AMÉRICA DEL SUR}

A nivel mundial están disponibles $13000 \mathrm{MMt}$ de biomasa anual, que se utilizan para alimentación animal, humana y energía, representando el $60 \%, 15 \%$ y $25 \%$ respectivamente (CEPAL, 2015a). La demanda creciente de biomasa ha generado impactos en la producción agrícola, debido a que existe el cuestionamiento de usar el suelo para la siembra de cultivos energéticos poniendo en riesgo la seguridad alimentaria de cada país (Sims \& Bassam, 2004). A pesar de aquello, esto representa una oportunidad para países de economías emergentes basadas en la agricultura, pecuaria y agroindustria como es el caso de ALC (CEPAL, 2015a), ya que por la diversidad de biomasa que tiene la región, representa oportunidades estratégicas para el desarrollo regional y local, además de permitir la diversificación de sus matrices productivas y energéticas (FAO, 2013).

En el año 2010, se generaron 5,1 toneladas per cápita de biomasa en toda ALC, en la cual se incluyeron cultivos primarios, residuos agrícolas, agropecuarios y madera (Schandl et al., 2016). Se estima que para el año 2050, el potencial energético de biomasa producida por ALC representará entre el 17 y $26 \%$ de la oferta mundial de energía, convirtiéndose en el principal productor de biomasa a nivel mundial (Razo et al., 2007). Con lo que respecta a América del Sur, esta ha venido desarrollando planes energéticos en los que se incluye el uso más eficiente de las fuentes renovables como la producción de biocombustibles (OLADE, 2016). Como resultado de esta implementación, las emisiones de $\mathrm{CO}_{2}$ en el año 2019 disminuyeron el $0,7 \%$ en comparación con el año anterior (BP, 2020).

\subsection{Biomasa disponible en países de América del Sur}

\section{Argentina $(A R G)$}

Es un país que dispone de recursos energéticos importantes, tanto renovables como no renovables (FAO, 1995). En los últimos años la actividad económica de este país ha estado inmersa en la explotación de minerales metálicos e industriales, lo que ha afectado directamente a la estabilidad de la biomasa (PNUMA, 2013). Dentro del plan nacional “Argentina innovadora 2020", uno de los principales objetivos es desarrollar diversos productos industriales como biopolímeros, componentes químicos y bioenergía a partir de materias primas como la soja, maíz y algodón (CEPAL, 2015a). En la cosecha de los años 2010-2011, se produjo 100,81 MMt de granos (soja, maíz, trigo, sorgo, girasol). De la soja y girasol se obtuvieron 8,22 MMt de aceites vegetales (FAO, 2013), por lo que Argentina en el año 2011 se convirtió en el principal exportador de biodiesel a nivel mundial, siendo unos de los destinos la Unión Europea (Roitman et al., 2011).

Con lo que respecta a biomasa para fines energéticos, este país ofertó 804 kTEP (kilo toneladas equivalentes de petróleo) de bagazo de caña de azúcar, 913 kTEP de leña, 1673 kTEP de aceites vegetales y $425 \mathrm{kTEP}$ de alcoholes vegetales en el año 2015 (Ministerio de energía y minería, 2016). Adicionalmente, es un país con un alto potencial en biomasa lignocelulósica, el cual produce aproximadamente 3,54 MMt/año de residuos forestales (podas frutales, olivo, aserraderos); 0,40 MMt/año de residuos de molienda y 3,50 MMt/año de residuos agrícolas (arroz, caña, maní, algodón, olivo) (Roitman et al., 2011). Se estima que existen alrededor de $143 \mathrm{MMt} / \mathrm{año}$ de biomasa leñosa fácilmente accesible, proveniente de bosques nativos e implantados en el país (FAO, 2009). Por último, en el año 2015 se generó 1,57 millones de $\mathrm{m}^{3}$ de residuos de madera (FAO, 2017).

\section{Bolivia (BOL)}

En este país la información con respecto a biomasa fue escasa, sin embargo Bolivia por su ubicación geográfica y los recursos energéticos con los que cuenta (entre ellos la biomasa), es un país que está encaminado a convertirse en uno de los principales ejes de suministro energético de América del Sur (FAO, 1995). En el año 2014, se obtuvo 7 485,04 kBEP (kilo barriles equivalentes al petróleo) de biomasa de bagazo de caña de azúcar, leña y residuo animal, aportando el 4,54\% de la energía primaria total (Sánchez, 2015). Además, en el año 2015 se produjeron $402000 \mathrm{~m}^{3}$ de residuos de madera (FAO, 2017).

\section{Brasil (BRA)}

Es el principal exportador de bioetanol en el mundo, gracias a que posee un gran potencial territorial y clima favorable (CEPAL, 2004; PNUMA, 2013). Cuenta con una alta producción de biomasa que es procesada dentro de su territorio, dándole un valor agregado. La utilización de biomasa sostenible para fines energéticos en el año 2012 representó el 25\% del consumo final de energía (CEPAL, 2016) y se estima que para el año 2020 el uso de la biomasa represente el $32 \%$ de la matriz energética brasileña (FAO, 2013). Mientras tanto, en el año 2015 se generó 79,09 MMt de leña, 19,15 MMt de melaza de caña de azúcar, 162,59 MMt de bagazo de caña de azúcar. y 17,19 millones de $\mathrm{m}^{3}$ de residuos de madera (Black et al., 2016; CEPAL, 2015b; FAO, 2017; MME, 2015, 2016). 


\section{Chile (CHI)}

Es el principal país exportador de minerales metálicos e industriales como el cobre, a pesar de aquello las exportaciones de biomasa han ido en aumento desde 1980 (PNUMA, 2013). Chile, cuenta con un alto potencial en biomasa forestal debido a sus extensas plantaciones de pino y eucalipto (Pontt, 2008). Dispone de 5,60 millones de ha (hectáreas) de bosques nativos productivos y 2,90 millones ha de plantaciones forestales, dando un total de 8,35 millones de ha de plantaciones comercialmente explotables (ArteagaPérez et al., 2015). De estas plantaciones se obtiene una biomasa forestal de 21,60 millones de ts/año (tonelada seca por año), generando un promedio de $4 \mathrm{MMt} / \mathrm{año}$ de leña (Marcos, 2012; Rios etal., 2013). Además, Chile dispone de aproximadamente $900 \mathrm{MMt} /$ año de biomasa para producir biogás, de los cuales comprenden 551,57 MMt/año de materia orgánica proveniente de las plantas de sacrificio de animales (avícola, vacuno, porcino) (D. M. Paneque, 2011). Por otra parte se producen 7,97 MMt/año de residuos agrícolas, en los que sobresalen residuos de cultivos de remolacha con 2,50 MMt/año, trigo con 1,80 MMt/año, maíz con 1,50 MMt/año y papa con 1,10 MMt/año (M. Paneque et al., 2011). A su vez, en el año 2015 se produjeron 1,92 millones de $\mathrm{m}^{3}$ de residuos de madera (FAO, 2017).

\section{Colombia $(\mathrm{COL})$}

En el año 2001, Colombia marcó su entrada a la nueva era de la bioenergía. En la Tabla 1 se muestra el potencial de biomasa de este país. Los sectores con mayor producción de biomasa en Colombia son el azucarero y palmero, que han incursionado en la cogeneración eléctrica. El potencial agrícola de Colombia es de 22 millones de ha (FAO, 2011a). En el año 2012 las destilerías utilizaron cerca de 0,37 MMt de azúcar crudo para producir bioetanol. Anualmente los ingenios azucareros producen $6 \mathrm{MMt}$ de bagazo de caña, provenientes de los 23 MMt de caña de azúcar.

Tabla 1. Potencial de biomasa en Colombia

\begin{tabular}{|c|c|c|c|}
\hline $\begin{array}{l}\text { Sector de } \\
\text { biomasa } \\
\text { residual }\end{array}$ & Producto & Tipo de residuo & $\begin{array}{r}\text { Producción } \\
\text { (MMt/año) }\end{array}$ \\
\hline \multirow{8}{*}{ Agrícola } & Arroz & Tamo y cascarilla & 6,28 \\
\hline & Banano & $\begin{array}{l}\text { Raquis, vástago y } \\
\text { rechazo }\end{array}$ & 11,55 \\
\hline & Café & Pulpa, cisco y tallo & 15,53 \\
\hline & Caña panelera & $\begin{array}{l}\text { Bagazo y hojas de } \\
\text { cogollo }\end{array}$ & 9,51 \\
\hline & Maíz & $\begin{array}{c}\text { Rastrojo, tusa y } \\
\text { capacho }\end{array}$ & 1,94 \\
\hline & Palma africana & Cuesco, fibra y raquis & 1,66 \\
\hline & Plátano & $\begin{array}{l}\text { Raquis, vástago y } \\
\text { rechazo }\end{array}$ & 20,41 \\
\hline & Caña de azúcar & Hojas, cogollo y bagazo & 15,53 \\
\hline \multirow{3}{*}{ Pecuario } & Avícola & Estiércol & 3,45 \\
\hline & Bobino & Estiércol & 99,17 \\
\hline & Porcino & Estiércol & 2,80 \\
\hline \multirow{2}{*}{$\begin{array}{l}\text { Sólidos } \\
\text { orgánicos } \\
\text { urbanos }\end{array}$} & $\begin{array}{l}\text { Centros de acopio y } \\
\text { plazas de mercado }\end{array}$ & - & 0,12 \\
\hline & Urbanos de poda & - & 0,04 \\
\hline
\end{tabular}

En el mismo año se tuvo una capacidad de 0,50 MMt de aceite de palma africana para la producción de biodiesel (FAO, 2013). Con lo que respecta a la biomasa agrícola, a nivel nacional se generan 82,42 MMt/año de residuos. La biomasa residual que produce el sector pecuario y urbano es de 105,42 $\mathrm{MMt} / \mathrm{año}$ y $0,17 \mathrm{MMt} /$ año respectivamente (Escalante et al., 2010). En el año 2015, este país generó $361.000 \mathrm{~m}^{3}$ de residuos de madera (FAO, 2017).

\section{Ecuador (ECU)}

Es un país con tradición agrícola y ganadera que genera gran cantidad de biomasa que puede ser aprovechada energéticamente (FAO, 2013). Esto se ve representado en la producción de energía primaria del año 2015, en el que se utilizaron 1,47 MMt de bagazo de caña y 0,72 MMt de leña para generación eléctrica (MCSE, 2016). En la Tabla 2, se indica el potencial biomásico que tiene Ecuador, el cual proviene de los sectores agrícolas, pecuarios, forestales y municipales. Con lo que respecta a biomasa residual del sector agrícola se generan aproximadamente 18,23 MMt/año de residuos. En el sector pecuario se producen 1,44 MMt/año de biomasa residual, en el sector forestal se originan 0,22 MMt/año y el sector urbano genera 1,70 MMt/año de desechos municipales (CEPAL, 2004; MEER, 2014). Se estima que a partir de residuos agrícolas como hojas de maíz, cascarilla de arroz, fruto de palma y cáscara de frutas se podría generar el $50 \%$ de la demanda de energía eléctrica nacional (CEPAL, 2004). Por otro lado, en el año 2015 se produjeron $158000 \mathrm{~m}^{3}$ de residuos de madera (FAO, 2017).

Tabla 2. Potencial biomásico en Ecuador

\begin{tabular}{|c|c|c|c|}
\hline $\begin{array}{l}\text { Sector de } \\
\text { biomasa } \\
\text { residual }\end{array}$ & Producto & Tipo de residuo & $\begin{array}{r}\text { Producción } \\
\text { (MMt/año) }\end{array}$ \\
\hline \multirow{10}{*}{ Agrícola } & Arroz & Pajilla y cáscara & 2,11 \\
\hline & Banano & Raquis, seudotallo, hojas y rechazo & 4,93 \\
\hline & Cacao & $\begin{array}{l}\text { Poda, mazorca, cáscara de } \\
\text { mazorca, raquis y rechazo de } \\
\text { producto }\end{array}$ & 2,02 \\
\hline & Café & $\begin{array}{l}\text { Poda, renovación de plantas, } \\
\text { cáscara y pulpa }\end{array}$ & 0,10 \\
\hline & $\begin{array}{l}\text { Caña de } \\
\text { azúcar }\end{array}$ & Tallos, hojas, bagazo & 0,79 \\
\hline & Maíz duro & Hojas, tallo y mazorcas & 0,43 \\
\hline & $\begin{array}{l}\text { Palma } \\
\text { africana }\end{array}$ & $\begin{array}{c}\text { Hojas, raquis, fibras, cascarilla de } \\
\text { nuez }\end{array}$ & 6,87 \\
\hline & Piña & Hojas, corona, cáscara y corazón & 0,12 \\
\hline & Palmito & $\begin{array}{l}\text { Hojas, despuntes, capas exteriores, } \\
\text { rechazo }\end{array}$ & 0,48 \\
\hline & Plátano & Hojas, seudotallo, raquis, rechazo & 0,37 \\
\hline \multirow{4}{*}{ Pecuario } & Avícola & Excretas & 0,51 \\
\hline & Porcino & Excretas & 0,06 \\
\hline & $\begin{array}{l}\text { Vacuno } \\
\text { (carne) }\end{array}$ & Excretas & 0,01 \\
\hline & $\begin{array}{l}\text { Vacuno } \\
\text { (leche) }\end{array}$ & Excretas & 0,86 \\
\hline Forestal & Forestal & $\begin{array}{c}\text { Ramas, corteza, raíces, aserrín y } \\
\text { astillas }\end{array}$ & 0,22 \\
\hline Municipales & Municipales & - & 1,70 \\
\hline \multicolumn{4}{|c|}{ Fuente: Adaptado de (CEPAL, 2004; MEER, 2014) } \\
\hline
\end{tabular}

La matriz energética de este país tiene un elevado componente de biomasa, ésta representó el 31,60\% de la producción de energía primaria del año 2015 (MOPC, 2016). En este mismo año se ofertaron 1 610,83 kTEP de leña, 700,30 kTEP de productos de caña y 584,14 kTEP de residuos agroforestales 
(cáscara de algodón, bagazo de caña, carozo de coco) (MOPC, 2016). En la Tabla 3, se indica la disponibilidad de biomasa de los principales cultivos de este país.

\begin{tabular}{cc} 
& \multicolumn{2}{c}{ Tabla 3. Disponibilidad de biomasa } \\
\cline { 2 - 3 } Cultivo & Residuo (MMt/año) \\
\hline Soja & 7,46 \\
Algodón & 0,53 \\
Girasol & 0,64 \\
Caña & 0,70 \\
Maní & 0,07 \\
Sésamo & 0,06 \\
Fuente: Adaptado de (Forster-Carneiro et al., 2013; Hiloidhari et al., 2014;
\end{tabular}

\section{Perú (PER)}

La agenda energética 2010-2040 de este país consolida a largo plazo la introducción a energías renovables (FAO, 2011b). Perú forma parte de los países que tienen una excesiva dependencia a la leña y falta de acceso a energías más eficientes y de mayor calidad (CEPAL, 2003, 2004). Solo en el año 2012 se ofertaron 83431 TJ (Tera Joule) de leña en comparación de 19430 TJ de bagazo de caña (Carrasco, 2015). A nivel nacional existen 272 MMt de biomasa, de las cuales $256 \mathrm{MMt}$ corresponden a leña y $16 \mathrm{MMt}$ a residuos derivados de actividades agrícolas, agroindustriales y madereras (FAO, 2011b).

Actualmente, existen 50201 ha de palma africana y 20000 ha de caña de azúcar que son destinadas para la producción de biocombustibles. De la utilización de caña de azúcar para bioetanol se dispone de 8,48 MMt de bagazo de caña (FAO, 2011b). Por otro lado, se calculó que para el año 2015 los residuos agrícolas de cultivos como la caña de azúcar, maíz amarillo duro, algodón, arroz y sorgo dulce aportaron 133455 TJ (FAO, 2011b).

\section{Uruguay (URU)}

En el año 2015, se ofertaron 0,27 MMt de residuos de caña de azúcar, sorgo dulce, soja, girasol, canola y sebo del sector agropecuario para la producción de biocombustibles (MIEM, 2015). Con lo que respecta a la biomasa residual agrícola se obtienen cada año aproximadamente 0,20 MMt de cáscara de arroz, 0,26 MMt de paja de trigo y 0,04 MMt de cáscara de girasol. Por otro lado, en el año 2015 se obtuvieron 2,16 MMt de leña (MIEM, 2015), 1,66 MMt de residuos forestales provenientes de poda y cosecha (DNETN, 2006) y $4000 \mathrm{~m}^{3}$ de residuos de madera (FAO, 2017).

\section{Venezuela (VEN)}

La biomasa utilizada en este país es casi marginal, debido principalmente a que es un país autoabastecido y exportador de hidrocarburos (CEPAL, 2003, 2004). A pesar de ello, desde el año 1960 comienza el desarrollo de centrales azucareras en este país (Dominguez et al., 2010). Según la Confederación de Asociaciones de Productores Agropecuarios, se generan a nivel nacional 0,03 MMt/año de residuos de café, 2,85 MMt/año de residuos de caña de azúcar, 0,28 MMt/año de residuos de arroz, 0,53 MMt/año de residuos de maíz (paja y tallos) y 0,17 MMt/año de residuos de sorgo (FEDEAGRO, 2016). Adicionalmente, en el año 2015 se produjeron 123000 $\mathrm{m}^{3}$ de residuos de madera (FAO, 2017). Se estima que la biomasa existente en Venezuela pueda proporcionar $740 \mathrm{kBEP}$ (Moreno, 2013).

\section{BIOPLÁSTICOS BIOBASADOS BIODEGRADABLES}

Los bioplásticos son la bioindustria de más rápido crecimiento a nivel global, atrayendo la principal atención de gobiernos e inversionistas (Lee, 2016). En el año 2016, a nivel mundial se produjeron 4,20 MMt de bioplásticos, de los cuales el 5,90\% fueron fabricados en América del Sur (European Bioplastics, 2017a). Para el año 2018 se estima que Asia, América del Sur y Europa sean los continentes con mayor capacidad de producción de bioplásticos con el 75,80\%, 12,20\% y 7,60\% respectivamente (Lee, 2016).

Las materias primas utilizadas para la producción de bioplásticos provienen principalmente de la biomasa residual del sector agrícola, como los residuos ricos en carbohidratos, azúcares y biomasa lignocelulósica (European Bioplastics, 2017b). En este sentido, en el año 2019 se utilizaron 0,79 millones de ha, lo que equivale al $0,01 \%$ de la superficie agrícola del mundo y se estima que para el año 2024 se necesite 1 millón de ha para obtener estas materias primas, representando el $0,021 \%$ de la superficie agrícola mundial (European Bioplastics, 2019).

Los BBB corresponden a los basados en almidón, proteína, celulosa, ácido poliláctico y producidos por microorganismos como se indica en la Figura 2 (European Bioplastics, 2016). En el año 2019, los BBB representaron el 55,5\% de la producción mundial de bioplásticos y se prevé que su capacidad aumente a 2,42 MMt para el año 2024 (European Bioplastics, 2019). Estos bioplásticos actualmente se utilizan en las industrias textiles, empaques rígidos y flexibles, dispositivos electrónicos y en la agricultura (European Bioplastics, 2019). Esto se debe a que se pueden procesar utilizando las mismas tecnologías (extracción, soplado o inyección) de los termoplásticos convencionales (ValeroValdivieso et al., 2013), sin embargo la fabricación de estos presenta algunos problemas con respecto con las materias primas, consumo de energía y costos operacionales (Ramesh et al., 2017). Los BBB como el ácido poliláctico (PLA), polihidroxialcanoatos (PHA's) o los basados en almidón ofrecen propiedades mejoradas como la flexibilidad, durabilidad, imprimibilidad, transparencia, resistencia al calor y brillo (European Bioplastics, 2017b). 



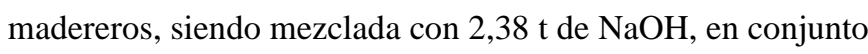

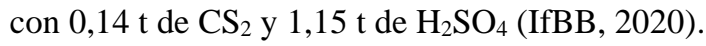

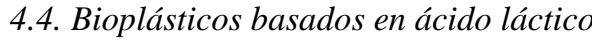

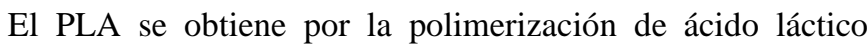

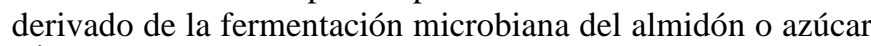

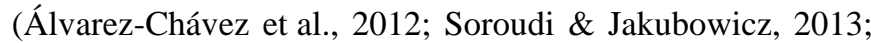

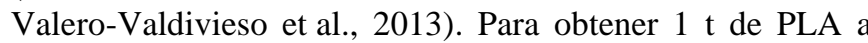

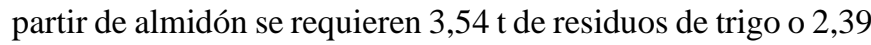

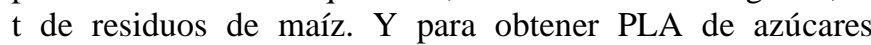

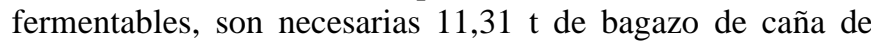

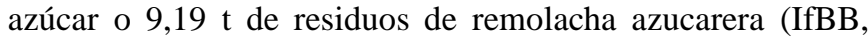

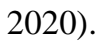

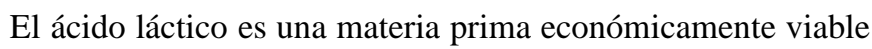

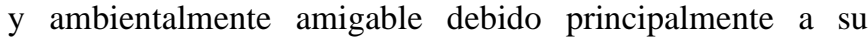

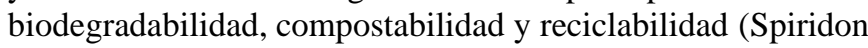

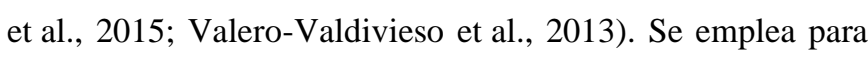

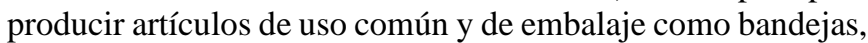

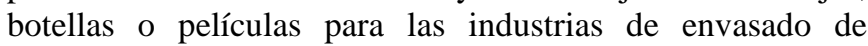

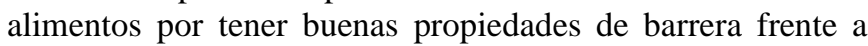

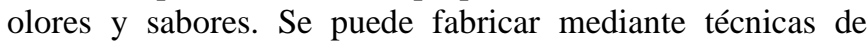

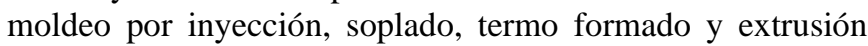

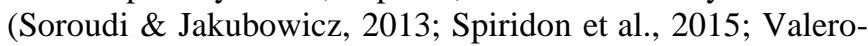

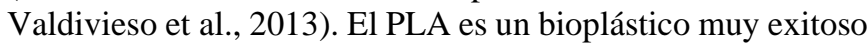

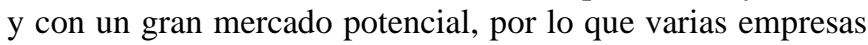

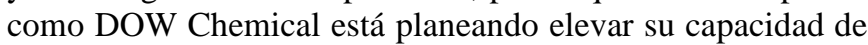

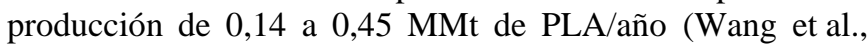

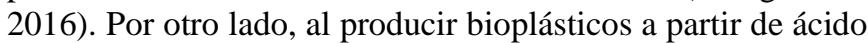

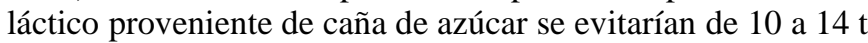

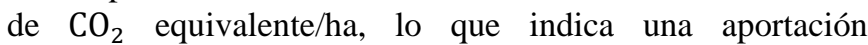

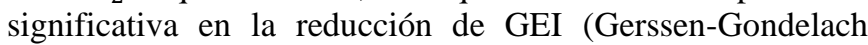

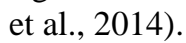

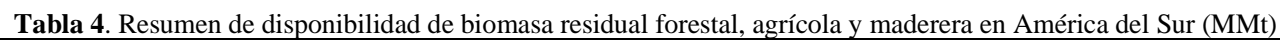

\begin{tabular}{|c|c|c|c|c|c|c|c|c|c|c|c|}
\hline 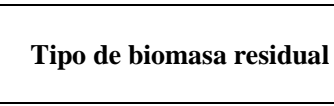 & 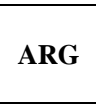 & 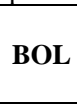 & 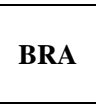 & 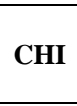 & 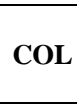 & 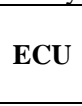 & 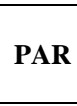 & 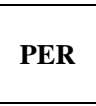 & 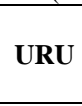 & 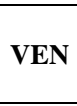 & 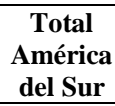 \\
\hline 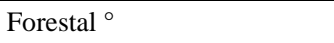 & 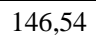 & 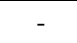 & 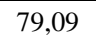 & 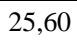 & 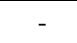 & 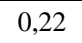 & 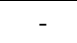 & 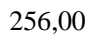 & 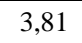 & 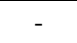 & 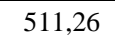 \\
\hline 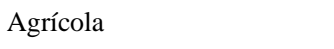 & 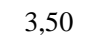 & 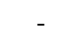 & 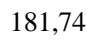 & 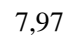 & 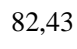 & 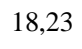 & 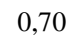 & 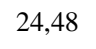 & 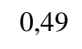 & 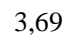 & 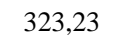 \\
\hline 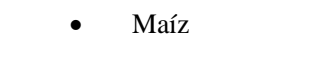 & 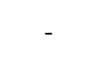 & 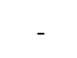 & 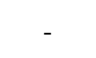 & 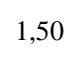 & 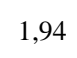 & 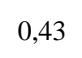 & 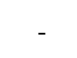 & 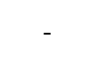 & 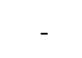 & 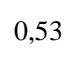 & 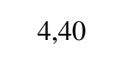 \\
\hline 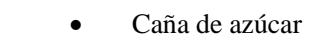 & 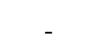 & 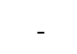 & 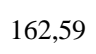 & 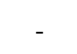 & 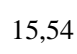 & 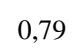 & 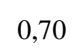 & 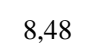 & 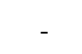 & 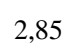 & 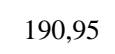 \\
\hline 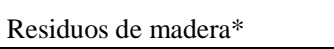 & 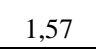 & 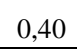 & 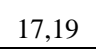 & 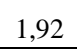 & 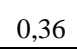 & 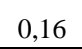 & 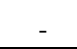 & 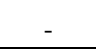 & 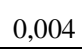 & 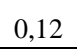 & 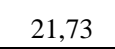 \\
\hline
\end{tabular}

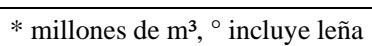

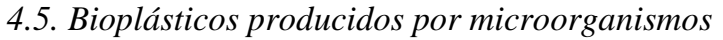

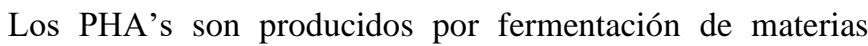

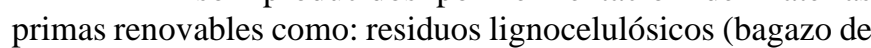

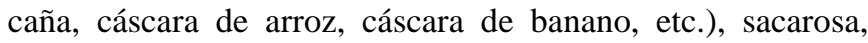

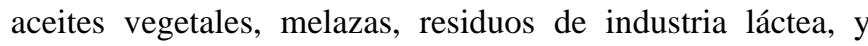

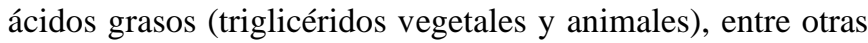

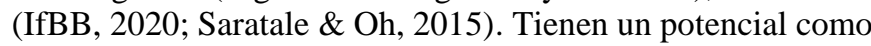

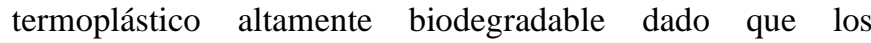

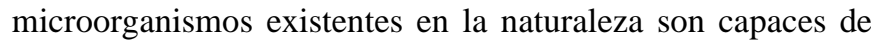

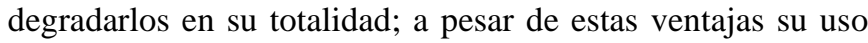

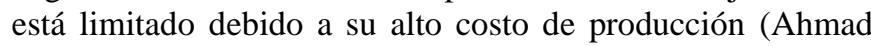

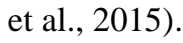

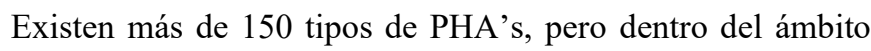

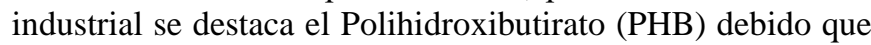

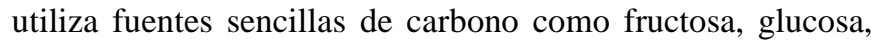

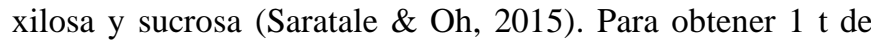

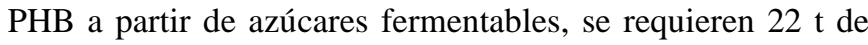

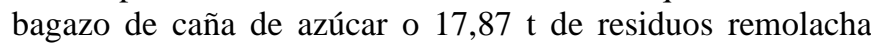

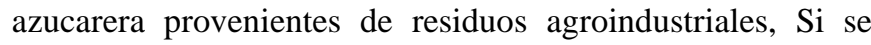

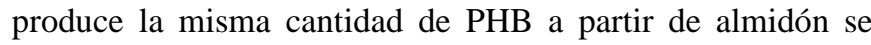

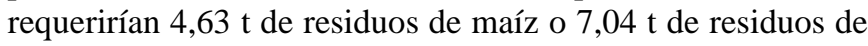

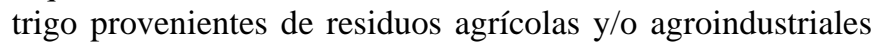

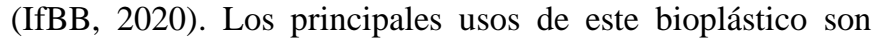

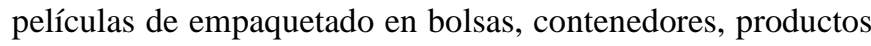

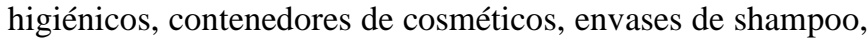

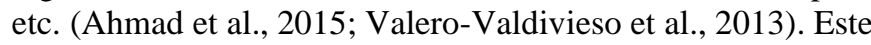

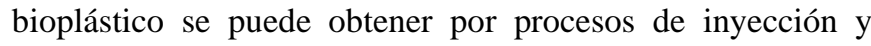

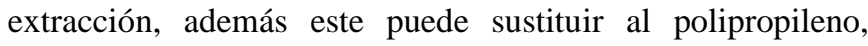

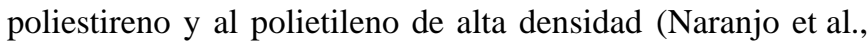

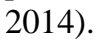

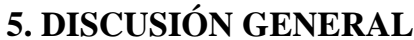

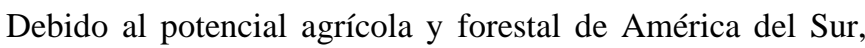

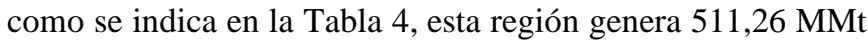

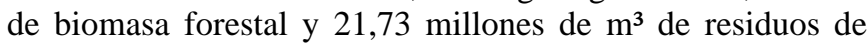

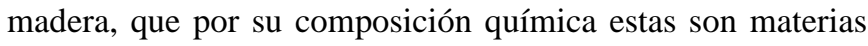

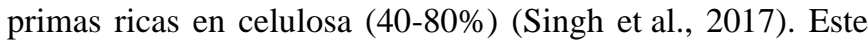

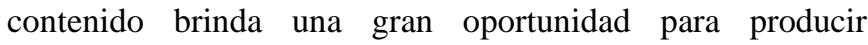

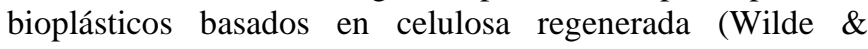

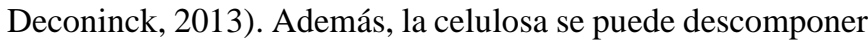

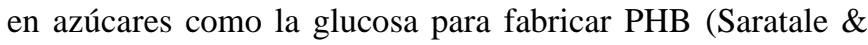

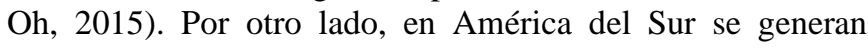

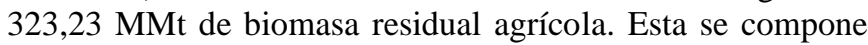

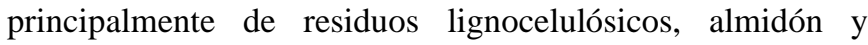

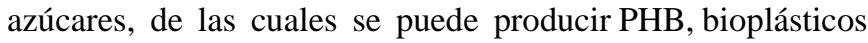

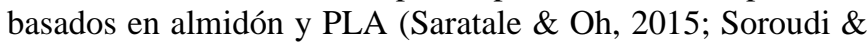

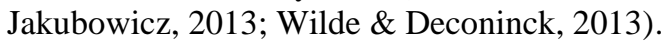

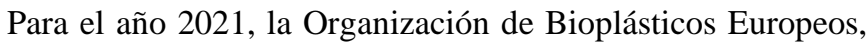

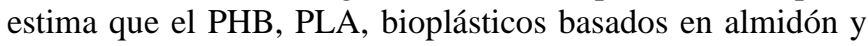

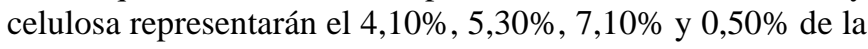

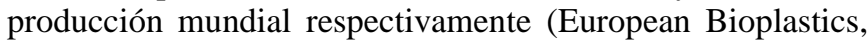

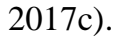

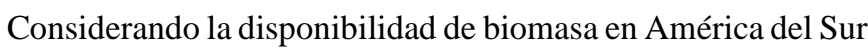

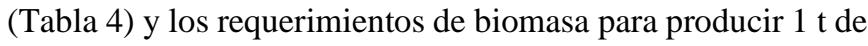

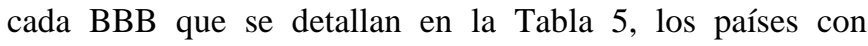

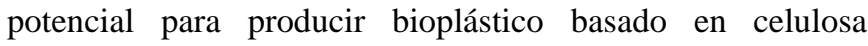


regenerada a partir de biomasa forestal son: Argentina, Brasil, Chile, Perú y Uruguay, los cuales producirían un total de 204,42 MMt/año de este bioplástico.

Tabla 5. Requerimiento de biomasa para obtener 1 tonelada de cada tipo de BBB

\begin{tabular}{ccc}
\hline Tipo de BBB & Tipo de biomasa & $\begin{array}{c}\text { Requerimiento de } \\
\text { biomasa* }\end{array}$ \\
\hline $\begin{array}{c}\text { Basados en almidón } \\
\text { Basados en celulosa } \\
\text { regenerada }\end{array}$ & Residuos de maíz & 1,07 \\
PHB & Forestal & 2,50 \\
PLA & azúcar & 21,99 \\
\hline Rn toniduos de trigo & 3,54
\end{tabular}

Adaptado de: (IfBB, 2020)

Por otro lado, Chile, Colombia, Ecuador y Venezuela tienen el potencial de fabricar 1,40 MMt/año, 1,81 MMt/año, 0,41 MMt/año y 0,50 MMt/año de bioplástico basado en almidón respectivamente, teniendo en cuenta el requerimiento de biomasa de residuos de maíz. Con respecto al PHB, considerando los valores disponibles de bagazo de caña y que se requieren 21,99 t de biomasa proveniente de dicho cultivo, se pueden producir 7,39 MMt/año, 0,71 MMt/año, 0,04 MMt/año, 0,03 MMt/año, 0,39 MMt/año y 0,13 MMt/año de PHB en Brasil, Colombia, Ecuador, Paraguay, Perú y Venezuela respectivamente. Además, a partir de los residuos disponibles de trigo se pueden producir 0,51 MMt/año y 0,07 MMt/año de PLA en Chile y Uruguay respectivamente, considerando los datos indicados en la Tabla 4.

En la Figura 3, se indica la capacidad de producción de BBB en América del Sur a partir de la disponibilidad de biomasa residual por país, en el cual se observa que Perú es el país con mayor potencial para producir bioplástico basado en celulosa regenerada debido a la dependencia y disponibilidad de biomasa forestal y residuos de madera. A su vez, se identifica que Chile puede obtener tres tipos de BBB, brindándole la oportunidad de diversificar su producción y convertirse en una potencia de América del Sur por la variedad y disponibilidad de biomasa que posee.

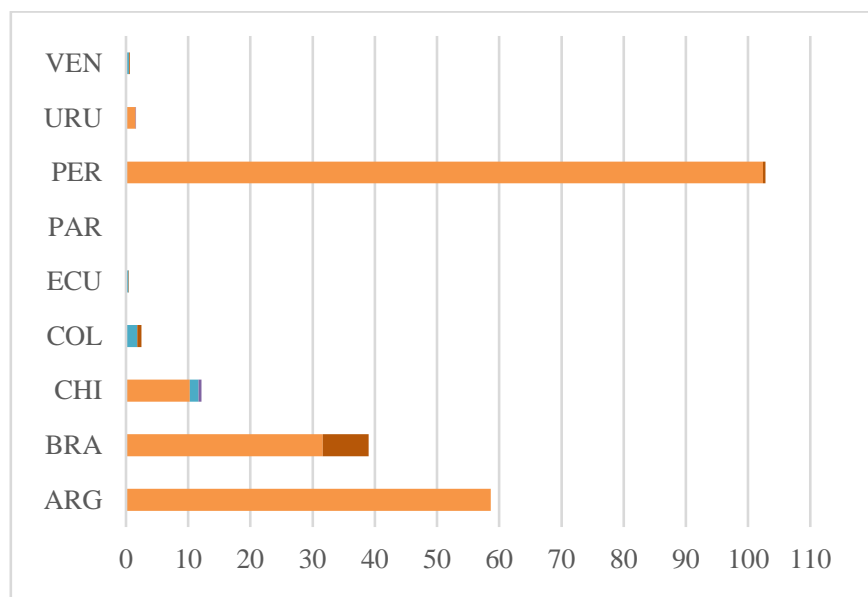

$\square$ Basados en celulosa regenerada $₫$ Basados en Almidón $\square$ PLA $\square$ PHB

Figura 3. Distribución de la producción de BBB en América del Sur, en $\mathrm{MMt}$
Con respecto a las emisiones de GEI al producir PLA y PHB a partir de maíz, se reducirían de 3 a $14 \mathrm{tCO}_{2}$ equivalente/ha y de 0 a $7 \mathrm{tCO}_{2}$ equivalente/ha respectivamente. Mientras que las emisiones de GEI se reducirían entre 10 a $14 \mathrm{tCO}_{2}$ equivalente/ha para el PLA y 3 a $14 \mathrm{tCO}_{2}$ equivalente/ha en la producción de PHB utilizando caña de azúcar como materia prima (Gerssen-Gondelach et al., 2014). Esto brinda a Brasil, Colombia, Ecuador, Paraguay, Perú y Venezuela la ventaja de distribuir sus residuos de bagazo de caña para producir PLA y $\mathrm{PHB}$, aportando con la reducción de emisiones de GEI.

Además de los residuos agrícolas mencionados, es importante considerar que varios países de América del Sur son potencias mundiales en la exportación de granos, frutas y/o flores como es el caso de Ecuador. Este país es el principal exportador de banano a nivel mundial (Redagrícola, 2020). En el año 2019 se produjeron 6,58 MMt de banano, lo que a su vez generó 0,07 MMt de banano de rechazo (INEC, 2020), que en la mayoría de los casos son arrojados al aire libre, generando un problema ambiental (Moreira Carrión, 2013; Ramírez \& Solórzano, 2012). Este residuo puede ser utilizado para producir bioplásticos como el PHB y PLA, debido a que la pulpa y la cáscara contienen almidón y azucares respectivamente (Naranjo et al., 2014).

Considerando lo mencionado anteriormente, existe un gran potencial para que la industria del plástico de América del Sur desarrolle nuevas líneas de productos en relación a los BBB. Sin embargo, estas industrias deberán enfrentar desafíos en investigación y desarrollo de nuevos procesos (Iles \& Martin, 2013). Para lo cual, será necesario generar vínculos con centros de investigación, universidades e institutos que permitan establecer estrategias de I+D+i (Investigación, Desarrollo e Innovación) necesarias para cumplir con las proyecciones de cada industria y reducir la contaminación que generan los plásticos sintéticos. Cabe recalcar que en América del Sur existen empresas que actualmente producen envases con PLA como es el caso de Vaiv (Chile) (Vaiv, 2015), Zeaplast (Chile) (ZeaPlast, 2012) y Grupo Phoenix (Colombia, Venezuela, Brasil, Uruguay y Ecuador) (Grupo Phoenix, 2014), las cuales aportan al cambio de la matriz productiva de la región y al fomento de plazas de trabajo en zonas rurales.

Por otro lado, si los gobiernos de cada país de América del Sur siguen intensificando la bioeconomía de los bioplásticos, traerán consigo varios beneficios como la menor dependencia de los recursos fósiles y desarrollo de varias industrias sostenibles (Lee, 2016). Para que esta transición no genere perturbaciones, es necesario establecer nuevas estrategias de producción en los que se utilicen una mínima cantidad de recursos como el agua, agroquímicos y fertilizantes. Esto a su vez debe brindar beneficios a la sociedad como seguridad alimentaria, energética y una distribución equitativa de los recursos (Sleenhoff et al., 2015). Así también el mercado de los BBB debe superar barreras relacionadas al uso de la tierra para producir cultivos con fines no alimentarios; el acceso restringido a los residuos biomásicos y sobre todo superar obstáculos mercantiles con sectores más establecidos en varios países como es el caso de los biocombustibles, los cuales disponen de un beneficio legal preferencial perjudicando el sector de los bioplásticos (Morone et al., 2015). Por todo lo 
mencionado, resulta imperante desarrollar vínculos entre la economía circular, bioeconomía y los BBB para estimular la innovación y la comercialización de este tipo de productos biobasados (European Commission, 2017).

Finalmente, en América del Sur se deben buscar las formas necesarias para contrarrestar la desigualdad social, especialmente la que se genera en zonas rurales y que por cuestiones políticas o por carencia de planificación no ha sido mitigada (Akella et al., 2009). Por otra parte, varios investigadores (Akella et al., 2009; Bioenarea, 2013) señalan que estas zonas podrían tener un desarrollo sostenible mediante el fomento de la bioeconomía zonal.

\section{CONCLUSIÓN}

La presente investigación reveló que en América del Sur se puede producir 204,42 MMt de bioplástico basado en celulosa regenerada, 4,11 MMt de bioplástico basado en almidón, además 0,58 MMt de PLA y 8,68 MMt de PHB, debido a la disponibilidad de biomasa que tiene la región y considerando los requerimientos de biomasa para la producción de $1 \mathrm{t}$ de los diferentes tipos BBB. Por otra parte, se pudo connotar que, si se aprovecha esta biomasa con fines energéticos y/o no energéticos, esta región puede pasar de una economía dependiente de recursos fósiles a una economía basada en biomasa y dejar atrás la histórica designación de ser exportadora de materias primas.

Adicionalmente, la producción de bioplásticos basados en biomasa residual es una alternativa para contrarrestar la contaminación ambiental producida por los plásticos sintéticos, ya que se aprovecha de una manera eficiente y sostenible los residuos que se generan en la naturaleza y en las industrias. Por otro lado, cada país de América del Sur deberá evaluar y determinar las mejores condiciones de recolección, transporte, almacenamiento, transformación de la biomasa y entrega de los bioplásticos, debido principalmente a que estos factores son determinantes en la evaluación de los costos de inversión y operación de este tipo de bioindustrias. Con base en esto, es fundamental y se recomienda que las instituciones de educación superior enfoquen la I+D+i a estos desafíos, en donde se incluya la evaluación de las materias primas, rutas de procesamiento, aspectos logísticos, sociales, ambientales, políticos y legales en cada una de las etapas de la ingeniería conceptual, básica y detalle, con el fin de aportar en la diversificación de las matrices productivas y energéticas, además de contribuir al desarrollo regional respondiendo a los problemas globales como la equidad, desarrollo sostenible, garantía de suministro de energía, empleo y mitigación del cambio climático.

\section{AGRADECIMIENTOS}

Los autores agradecen al Instituto de Fomento al Talento Humano de Ecuador (IFTH) por el apoyo financiero otorgado para realizar esta investigación.

\section{REFERENCIAS}

Ahmad, M., Mohd, K., Arif, H., Noriznan, M., Salihon, J., Shirai, Y., \& Ali, M. (2015). Case study for a palm biomass biore fi nery utilizing renewable non-food sugars from oil palm frond for the production of poly ( 3-hydroxybutyrate ) bioplastic. Journal of Cleaner Production, 87, 284-290. https://doi.org/10.1016/j.jclepro.2014.10.010

Akella, A. K., Saini, R. P., \& Sharma, M. P. (2009). Social, economical and environmental impacts of renewable energy systems. Renewable Energy, 34(2), 390-396. https://doi.org/10.1016/j.renene.2008.05.002

Almeida, A. de, Ruiz, J. A., López, N. I., \& Pettinari, M. J. (2004). Bioplásticos: una alternativa ecológica. Quimica Viva, 3, 1-24. https://doi.org/10.1111/tpj.12777/1/24

Álvarez-Chávez, C. R., Edwards, S., Moure-Eraso, R., \& Geiser, K. (2012). Sustainability of bio-based plastics : general comparative analysis and recommendations for improvement. Journal of Cleaner Production, 23(1), 47-56. https://doi.org/10.1016/j.jclepro.2011.10.003

Ambientum.com. (2015). Energía - Cultivos energéticos. Portal profesional del medio ambiente. http://www.ambientum.com/enciclopedia medioambiental/energia/C ultivos_energeticos.asp

Arteaga-Pérez, L. E., Vega, M., Rodríguez, L. C., Flores, M., Zaror, C. A., \& Casas Ledón, Y. (2015). Life-Cycle Assessment of coal-biomass based electricity in Chile: Focus on using raw vs torrefied wood. Energy for Sustainable Development, 29, 81-90. https://doi.org/10.1016/j.esd.2015.10.004

Berndes, G., Hoogwijk, M., \& Van Den Broek, R. (2003). The contribution of biomass in the future global energy supply: A review of 17 studies. Biomass and Bioenergy, 25(1), 1-28. https://doi.org/10.1016/S09619534(02)00185-X

Bioenarea. (2013). Biorrefinerías una oportunidad de negocio para las zonas

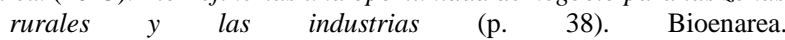
https://docplayer.es/27400896-Biorrefinerias-una-oportunidad-denegocio-para-las-zonas-rurales-y-las-industrias.html

Black, M. J., Sadhukhan, J., Day, K., Drage, G., \& Murphy, R. J. (2016). Developing database criteria for the assessment of biomass supply chains for biorefinery development. Chemical Engineering Research $\begin{array}{lll}\text { and Design, } & \text { 107, 253-262. }\end{array}$ https://doi.org/10.1016/j.cherd.2015.10.046

BP. (2020). Statistical Review of World Energy (Statistical Review of World $\begin{array}{lllll}\text { Energy (ed.); } & 69 \mathrm{a} \quad \text { ed.). }\end{array}$ https://www.bp.com/content/dam/bp/businesssites/en/global/corporate/pdfs/energy-economics/statisticalreview/bp-stats-review-2020-full-report.pdf

Carrasco, S. (2015). Balance de Energía Nacional, desde la perspectiva de supervisor.

https://www.osinergmin.gob.pe/seccion/centro_documental/hidrocarb uros/Publicaciones/BALANCE DE ENERGÍA EN EL PERU 2014.pdf

CEPAL. (2003). Sostenibilidad energética en América Latina y el Caribe: El aporte de las fuentes renovables. Comisión Económica para América Látina y el Caribe. http://repositorio.cepal.org/bitstream/handle/11362/2764/1/S2003717 _es.pdf

CEPAL. (2004). Fuentes renovables de energía en America Latina y el Caribe (pp. 1-159). Comisión Económica para América Látina y el Caribe Deutsche Gesellschaft für Technische Zusammenarbeit. http://www.cepal.org/publicaciones/xml/9/14839/lc12132e.pdf

CEPAL. (2006). Fuentes renovables de energía en América Latina y el Caribe, dos años despues de la conferencia de Bonn. Comisión Económica para América Látina y el Caribe. http://www.cepal.org/ilpes/noticias/paginas/8/35988/Fuentes_deEner giaRenovablenALClcw 100.pdf 


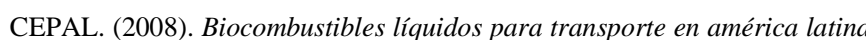

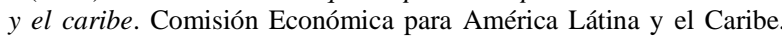

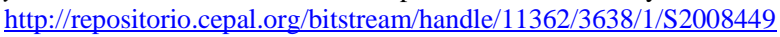

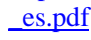

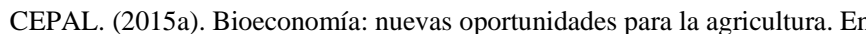

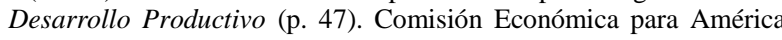

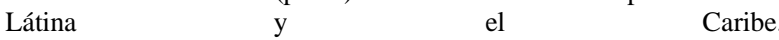

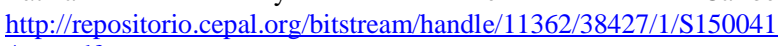

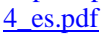

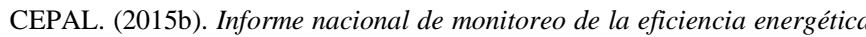

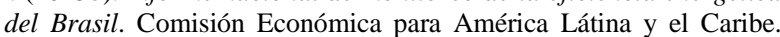

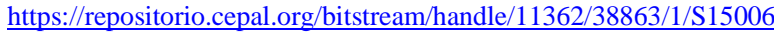

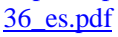

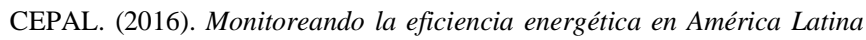

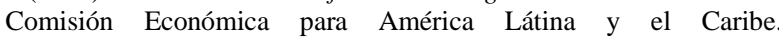

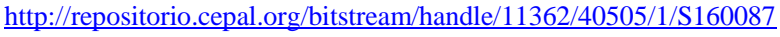

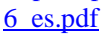

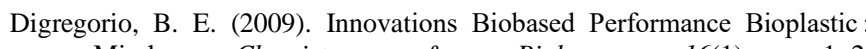

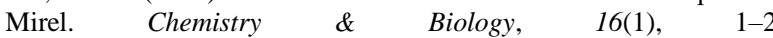

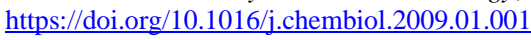

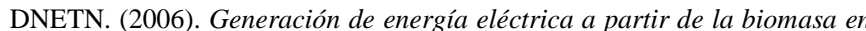

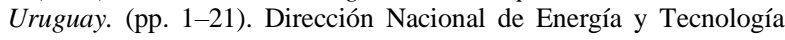

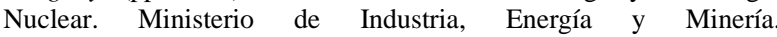

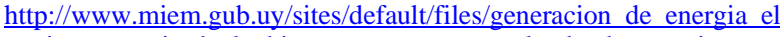

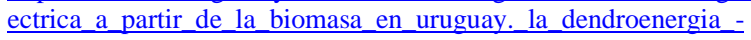

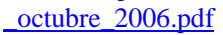

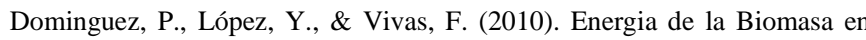

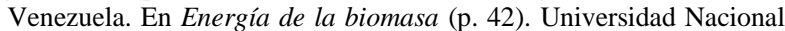

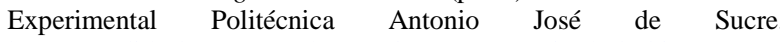

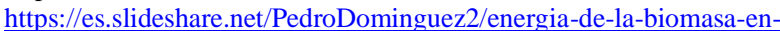

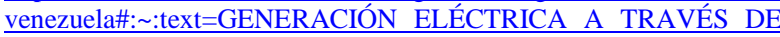

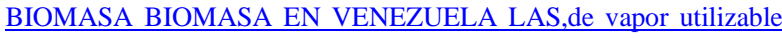

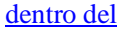

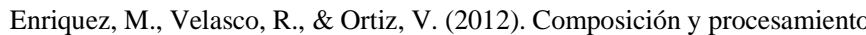

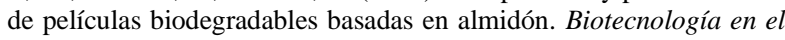

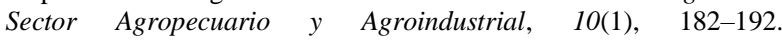

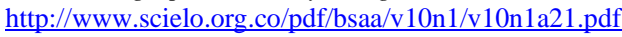

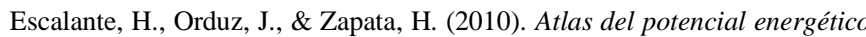

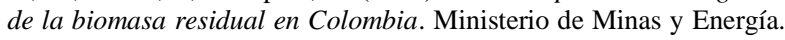

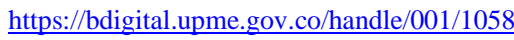

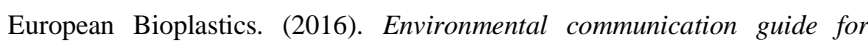

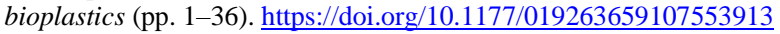

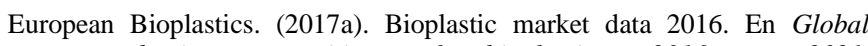

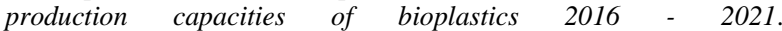

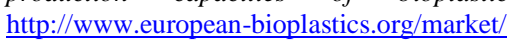

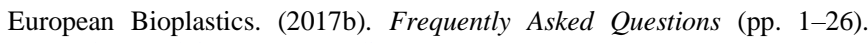

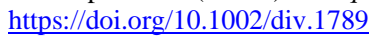

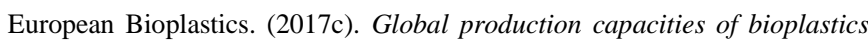

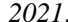

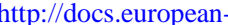

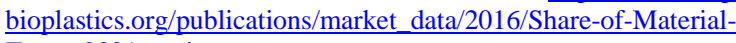

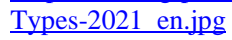

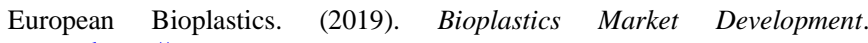

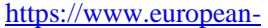

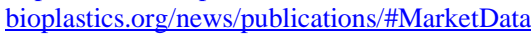

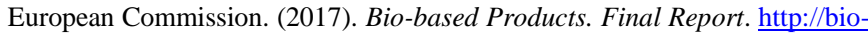

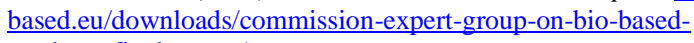

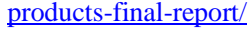

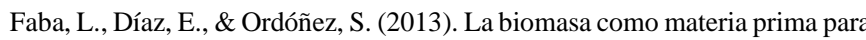

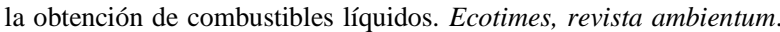

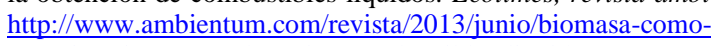

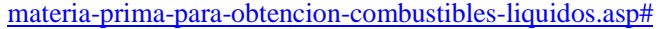

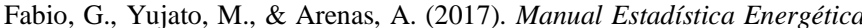

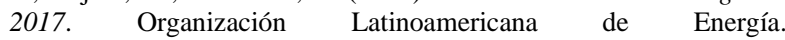

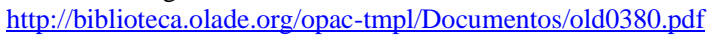

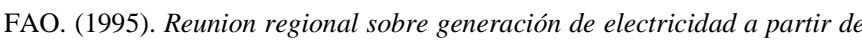

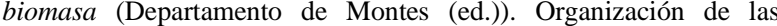

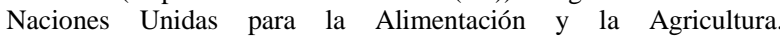

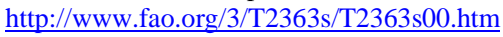

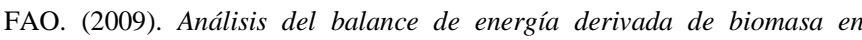

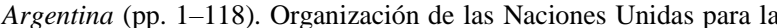

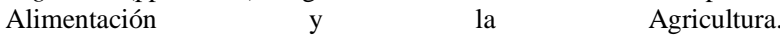

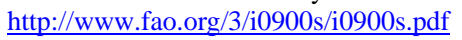

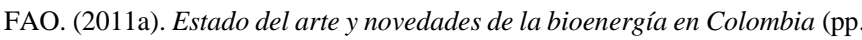

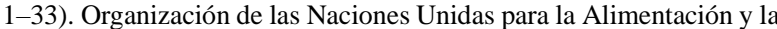

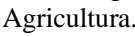

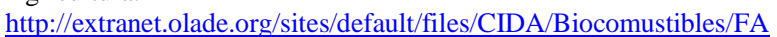

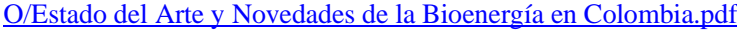

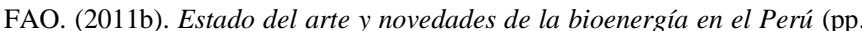

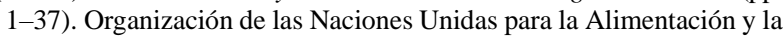

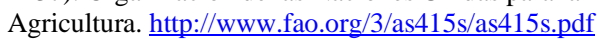

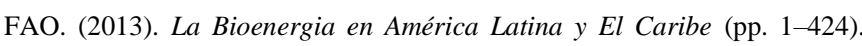

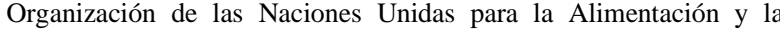

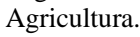

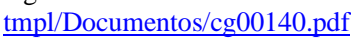

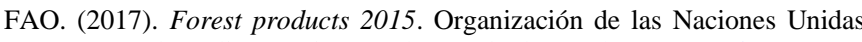

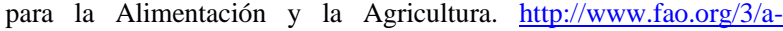

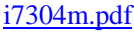

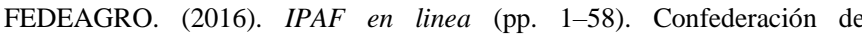

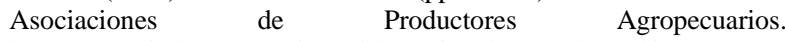

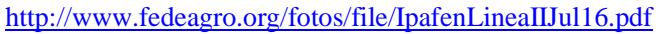

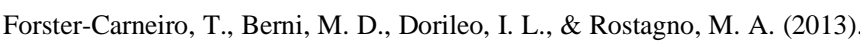

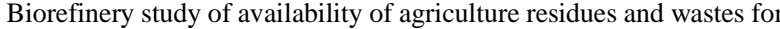

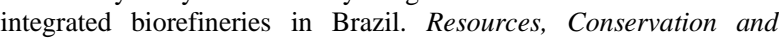

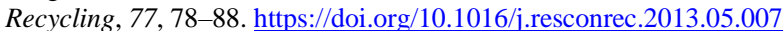

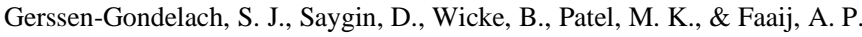

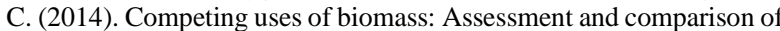

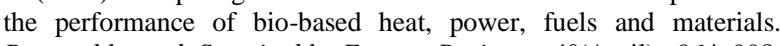

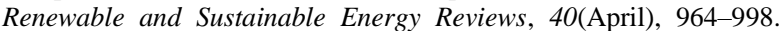

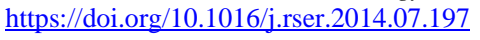

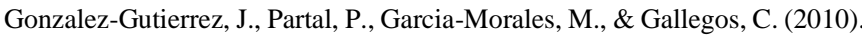

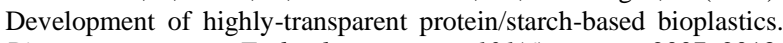

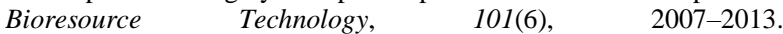

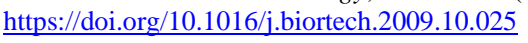

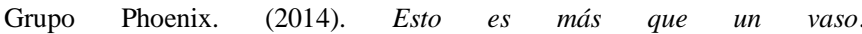

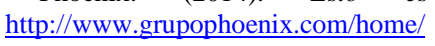

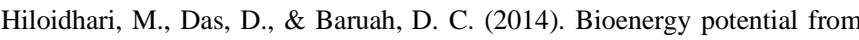

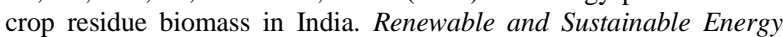

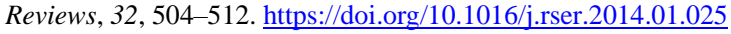

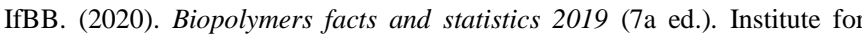

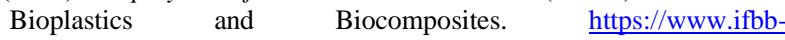

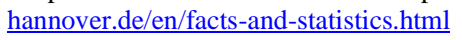

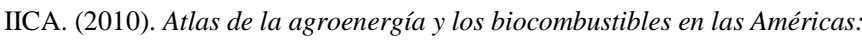

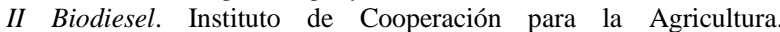

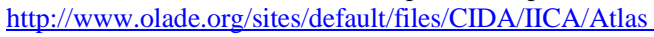

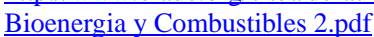

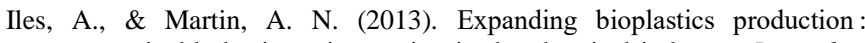

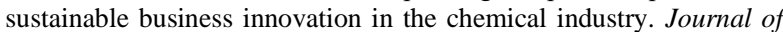

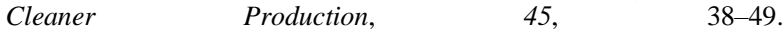

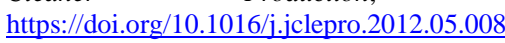

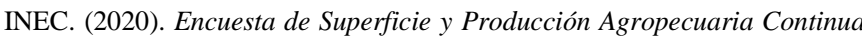

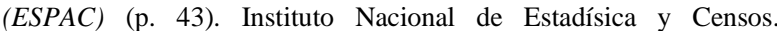


https://www.ecuadorencifras.gob.ec/documentos/webinec/Estadisticas_agropecuarias/espac/espac-2019/Presentacion de los principales resultados ESPAC 2019.pdf

Jerez, A., Partal, P., Martínez, I., Gallegos, C., \& Guerrero, A. (2007). Egg white-based bioplastics developed by thermomechanical processing. Journal of Food Engineering, 82(4), 608-617. https://doi.org/10.1016/j.jfoodeng.2007.03.020

Lee, D. H. (2016). Bio-based economies in Asia: Economic analysis of development of bio-based industry in China, India, Japan, Korea, Malaysia and Taiwan. International Journal of Hydrogen Energy, 41(7), 4333-4346. https://doi.org/10.1016/j.ijhydene.2015.10.048

Lovera, L. (2011). Estado del arte y novedades de la bioenergía en el Paraguay. Organización de las Naciones Unidas para la Alimentación y la Agricultura. http://www.fao.org/3/as414s/as414s.pdf

Marcos, S. (2012). Análisis económico-energético de la biomasa lignocelulósica en Chile [Pontificia Universidad Católica de Chile]. https://repositorio.uc.cl/handle/11534/1899

Martínez, I., Partal, P., García-morales, M., Guerrero, A., \& Gallegos, C. (2013). Development of protein-based bioplastics with antimicrobial

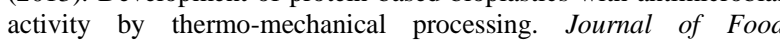
Engineering, $117,247-254$

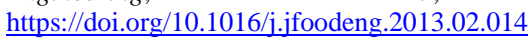

MCSE. (2016). Balance energético nacional 2016 - año base 2015 (Vol. 1

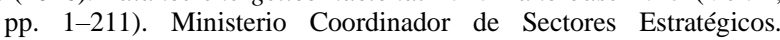
https://doi.org/10.1017/CBO9781107415324.004

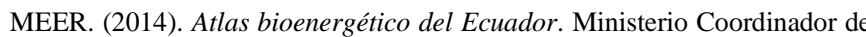
Producción, Empleo y Competitividad - Ministerio de Electricidad y

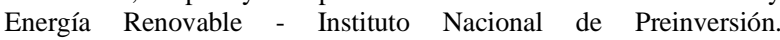
http://historico.energia.gob.ec/biblioteca/

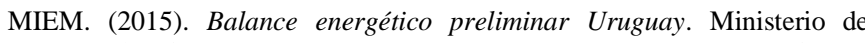
Industria, Energía y

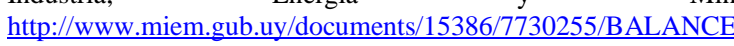
PRELIMINAR 2015.pdf

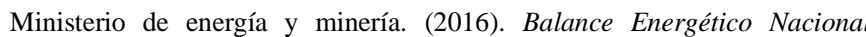

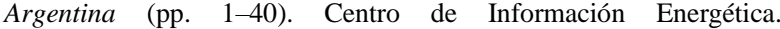

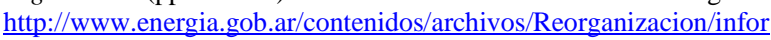

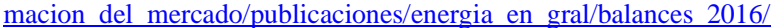

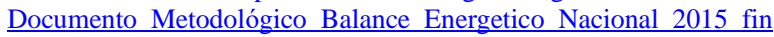

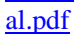

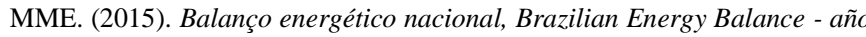

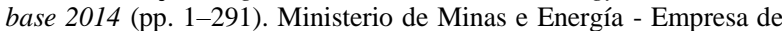

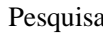

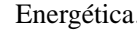

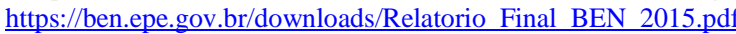

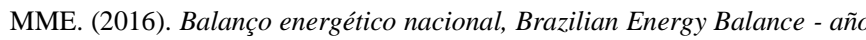

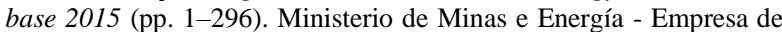

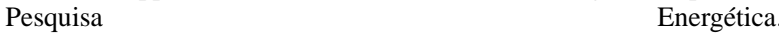

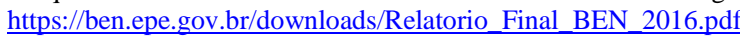

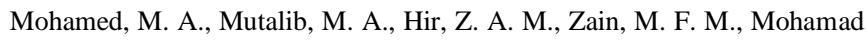

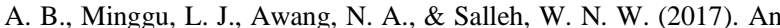

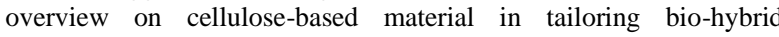

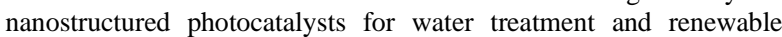

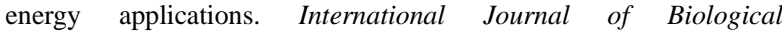

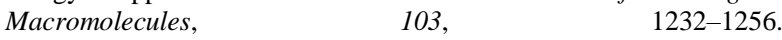

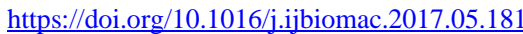

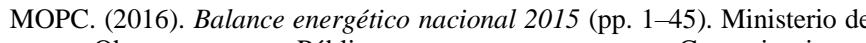

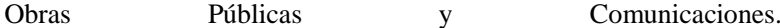

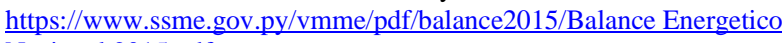

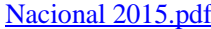

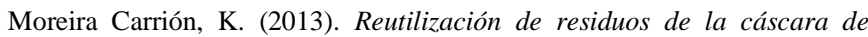

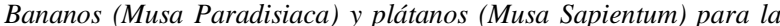

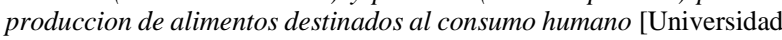

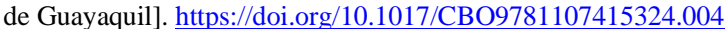

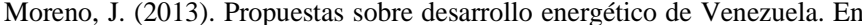

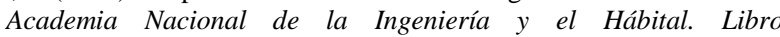

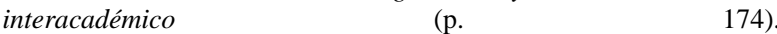

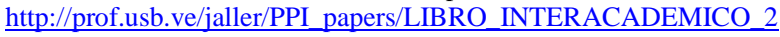

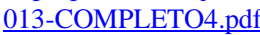

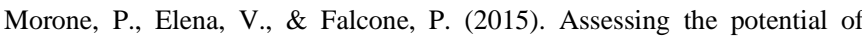

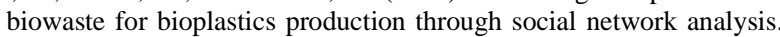

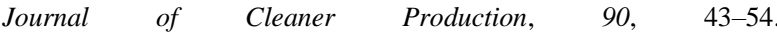

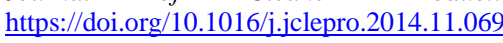

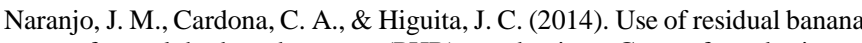

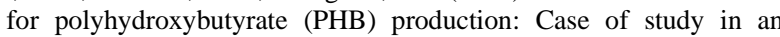

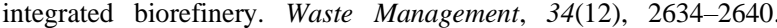

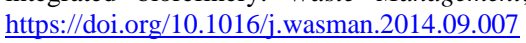

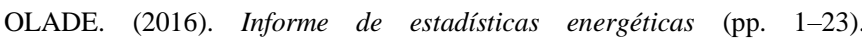

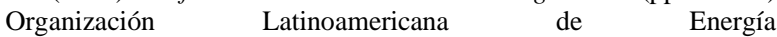

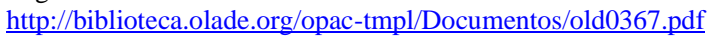

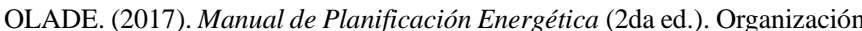

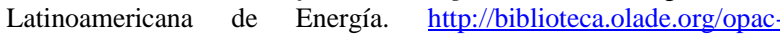

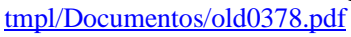

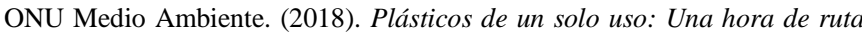

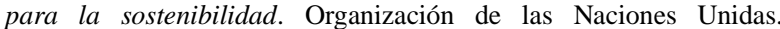

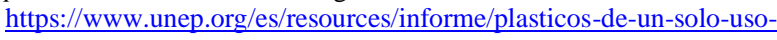

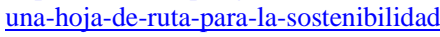

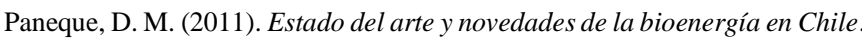

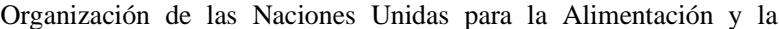

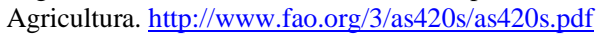

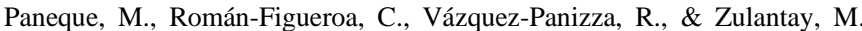

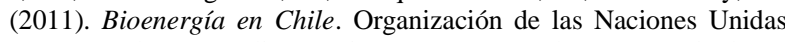

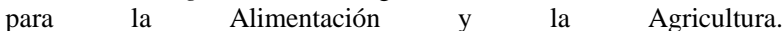

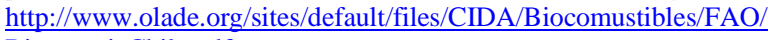

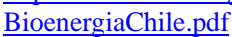

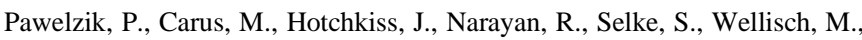

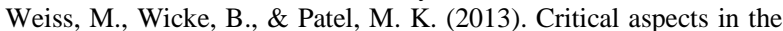

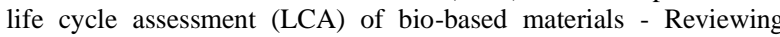

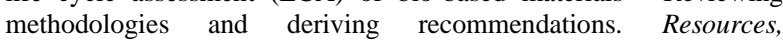

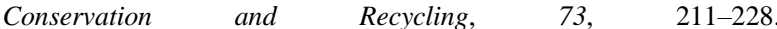

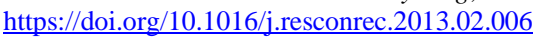

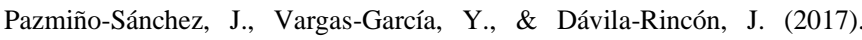

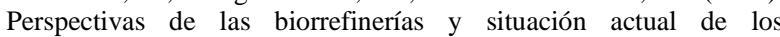

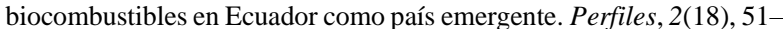

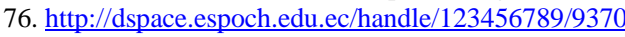

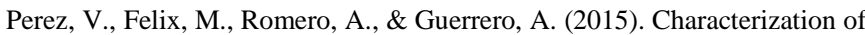

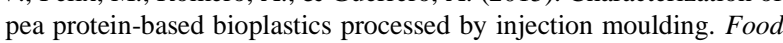

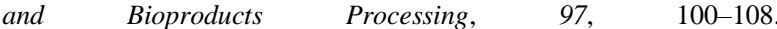

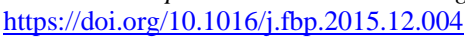

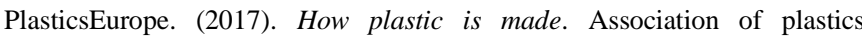

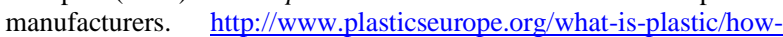

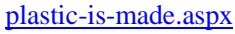

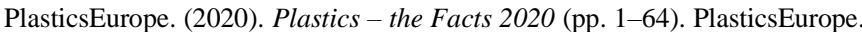

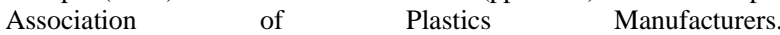

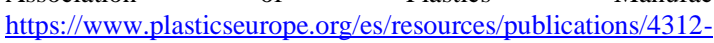

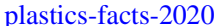

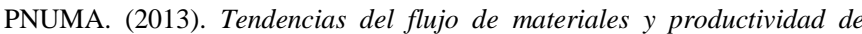

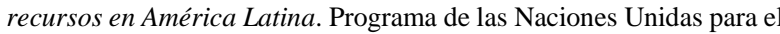

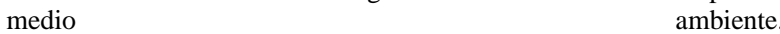

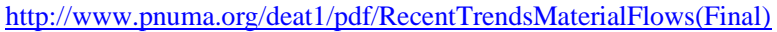

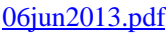

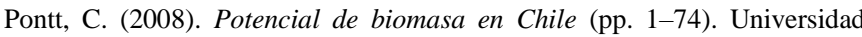

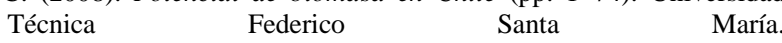

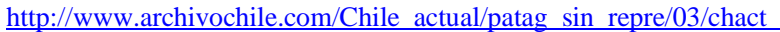

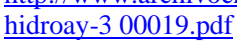


Prando, R. (2015). Biomasa, definición y características. Introducción a los procesos tecnológicos industriales, 1-121. https://es.scribd.com/document/424069803/EERR-Biomasa

Ramesh, M., Palanikumar, K., \& Reddy, K. H. (2017). Plant fibre based biocomposites: Sustainable and renewable green materials. Renewable and Sustainable Energy Reviews, 79(February), 558-584. https://doi.org/10.1016/j.rser.2017.05.094

Ramírez, J. C., \& Solórzano, S. A. (2012). Banano rechazado para exportación en Ecuador: propuesta de creación de valor para lograr su introducción al mercado internacional [Universidad Politécnica Salesiana

https://dspace.ups.edu.ec/bitstream/123456789/2840/1/UPSGT000315.pdf

Razo, C., Ludeña, C., \& Saucedo, A. (2007). Producción de biomasa para biocombustibles líquidos: el potencial de América latina y el Caribe. Comisión Económica para América Látina y el Caribe. http://repositorio.cepal.org/bitstream/handle/11362/4581/S0700830_e s.pdf? sequence $=1 \&$ is Allowed $=y$

Redagrícola. (2020). Colombia entre el "top 5" de los productores y exportadores de banana. https://www.redagricola.com/co/colombiaentre-el-top-5-de-los-productores-y-exportadores-de-banana/

Reddy, C. S. ., Ghai, R., Rashmi, \& Kalia, V. . (2003). Polyhydroxyalkanoates: an overview. Bioresource Technology, 87(2) 137-146. https://doi.org/10.1016/S0960-8524(02)00212-2

Rios, A., Almonacid, B., Holmqvist, C., \& Gutiérrez, D. (2013). Evaluación de Mercado de Biomasa y su Potencial - Versión Resumida. https://www.prevencionincendiosforestales.cl/documento/evaluacionde-mercado-de-biomasa-y-su-potencial/

Roitman, M., Martinelli, C., \& Rodriguez, C. (2011). Producción de energía a partir de biomasa. Energías alternativas de Córdoba, 1-34. http://www.epec.com.ar/docs/institucional/energiasalternativas/BIO MASA.pdf

Sánchez, L. (2015). Balance Energético Nacional Bolivia 2000-2014. Ministerio de Hidrocarburos y Energía. https://issuu.com/ministeriodehidrocarburos/docs/ben $2000 \quad 2014$

Santos, R. P. O., Rodrigues, B. V. M., Ramires, E. C., Ruvolo-Filho, A. C., \& Frollini, E. (2015). Bio-based materials from the electrospinning of lignocellulosic sisal fibers and recycled PET. Industrial Crops and Products, 72, 69-76. https://doi.org/10.1016/j.indcrop.2015.01.024

Saratale, G. D., \& Oh, M. K. (2015). Characterization of poly-3hydroxybutyrate (PHB) produced from Ralstonia eutropha using an alkali-pretreated biomass feedstock. International Journal of Biological Macromolecules, $\quad 80, \quad 627-635$. https://doi.org/10.1016/j.ijbiomac.2015.07.034

Schandl, H., Fischer-kowalski, M., West, J., Giljum, S., Dittrich, M., Eisenmenger, N., Geschke, A., Lieber, M., Wieland, H., Schaffartzik, A., Lenzen, M., Tanikawa, H., \& Miatto, A. (2016). Global Material Flows Resource and Productivity. https://www.resourcepanel.org/reports/global-material-flows-andresource-productivity-database-link

Sims, R. E., \& Bassam, N. El. (2004). Biomass and Resources. En Bioenergy Options for a Cleaner Environment (pp. 1-28). Elsevier. https://doi.org/10.1016/B978-008044351-5/50003-9

Singh, Y. D., Mahanta, P., \& Bora, U. (2017). Comprehensive characterization of lignocellulosic biomass through proximate, ultimate and compositional analysis for bioenergy production. Renewable Energy, 103, 490-500. https://doi.org/10.1016/j.renene.2016.11.039

Sleenhoff, S., Landeweerd, L., \& Osseweijer, P. (2015). Bio-basing society by including emotions. Ecological Economics, 116, 78-83. https://doi.org/10.1016/j.ecolecon.2015.04.011
Soroudi, A., \& Jakubowicz, I. (2013). Recycling of bioplastics , their blends and biocomposites: A review. European Polymer Journal, 49(10), 2839-2858. https://doi.org/10.1016/j.eurpolymj.2013.07.025

Spiridon, I., Leluk, K., Maria, A., \& Nicoleta, R. (2015). Composites : Part B Evaluation of PLA - lignin bioplastics properties before and after accelerated weathering. COMPOSITES PART B, 69, 342-349. https://doi.org/10.1016/j.compositesb.2014.10.006

Vaiv. (2015). Agua Mineral - Toma Agua No plástico. http://www.vaiv.cl/

Valero-Valdivieso, M., Ortegon, Y., \& Uscategui, Y. (2013). Biopolymers: Progress and Prospects. SciELO Colômbia, 181, 171-180. http://www.revistas.unal.edu.co/index.php/dyna/article/viewFile/206 $\underline{42 / 42269}$

Wang, R., Zhang, J., Kang, H., \& Zhang, L. (2016). Design, preparation and properties of bio-based elastomer composites aiming at engineering applications. Composites Science and Technology, 133, 136-156. https://doi.org/10.1016/j.compscitech.2016.07.019

Wilde, B. de, \& Deconinck, S. (2013). Benefits and challenges of bio- and oxo-degradable plastics. https://www.ows.be/wpcontent/uploads/2013/10/Final-Report-DSL-1_Rev02.pdf

Yu, J. (2007). Microbial production of bioplastics from renewable resources. En S.-T. Yang (Ed.), Bioprocessing for Value-Added Products from Renewable Resources (pp. 585-610). Elsevier B.V. https://doi.org/10.1016/B978-0-444-52114-9.50024-4

Zahari, M. A. K. M., Ariffin, H., Mokhtar, M. N., Salihon, J., Shirai, Y., \& Hassan, M. A. (2015). Case study for a palm biomass biorefinery utilizing renewable non-food sugars from oil palm frond for the production of poly(3-hydroxybutyrate) bioplastic. Journal of Cleaner Production, 87(C), 284-290. https://doi.org/10.1016/j.jclepro.2014.10.010

Zárate-Ramírez, L. S., Romero, A., Bengoechea, C., Partal, P., \& Guerrero, A. (2014). Thermo-mechanical and hydrophilic properties of polysaccharide / gluten-based bioplastics. Carbohydrate Polymers, 112, 24-31. https://doi.org/10.1016/j.carbpol.2014.05.055

ZeaPlast. (2012). ZEAplast: Plásticos biodegradables: http://www.zeaplast.cl/

Zhang, B., \& Wang, Y. (2013). Biomass processing, conversion and biorefinery. En Biomass Processing, Conversion and Biorefinery (pp. 1-457). $\quad$ http://www.scopus.com/inward/record.url?eid=2-s2.084892095504\&partnerID=tZOtx3y1

\section{BIOGRAFÍA}

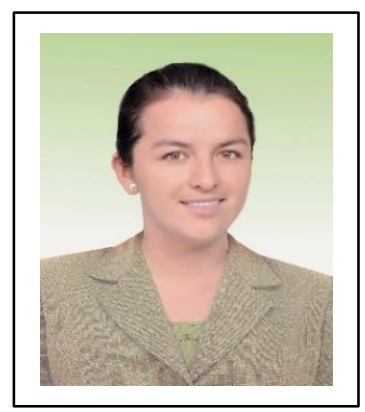

Yadira Vargas García, Ingeniera Industrial de la Escuela Superior Politécnica de Chimborazo, Magister en Ingeniería de Procesos y Sistemas Industriales graduada en la Universidad de Bogotá Jorge Tadeo Lozano. Profesionalmente vinculada a la docencia tecnológica superior e investigación en el diseño de procesos para obtención de bioplásticos dentro del contexto de biorrefinerías. 


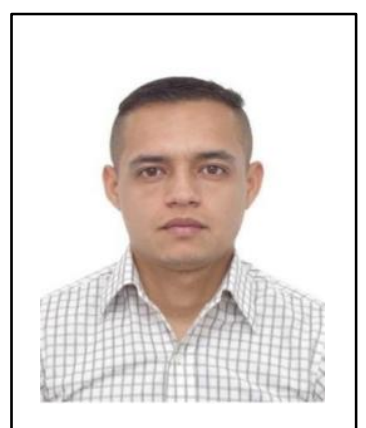

Joffre Pazmiño Sánchez, Ingeniero Industrial graduado de la Escuela Superior Politécnica de Chimborazo, Magíster en Ingeniería de Procesos y Sistemas Industriales de la Universidad de Bogotá Jorge Tadeo Lozano. Dentro del ámbito profesionalizante $\mathrm{e}$ investigativo labora en la formulación y evaluación de proyectos relacionados a la valorización e industrialización de la biomasa, gestión de la Seguridad, Salud y Ambiente, así como también en la optimización de procesos industriales. Adicional, es con fundador y asesor de la compañía SOPBIAL CÍA LTDA., la misma que participa en proyectos ambientales como industriales.

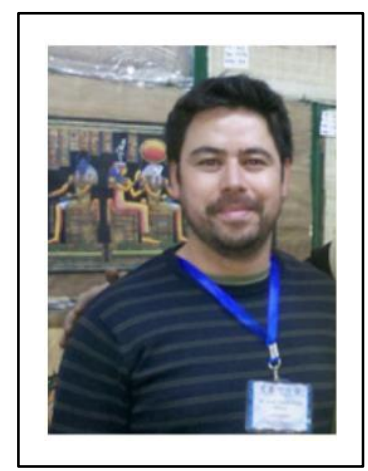

de cannabis medicinal.
Javier Dávila Rincón, Ingeniero químico egresado de la Universidad Nacional de Colombia, maestría en Ingeniería Química y $\mathrm{PhD}$ en Ingeniería. Trabaja en fluidos supercríticos para la extracción de aceites y biocompuestos así como en el diseño de procesos químicos y biorrefinerías. Aprovechamiento de residuos sólidos y productos derivados 